\title{
A correlation study of high-altitude and midaltitude clouds and galactic cosmic rays by MIPAS-Envisat
}

\author{
Susanne Rohs, ${ }^{1}$ Reinhold Spang, ${ }^{1}$ Franz Rohrer, ${ }^{2}$ Cornelius Schiller, ${ }^{1}$ and Heinz Vos ${ }^{1}$
}

Received 7 June 2009; revised 9 February 2010; accepted 5 March 2010; published 31 July 2010.

[1] The cloud index (CI), the cloud occurrence frequency (Occ), and the extinction data (Ext) of the Michelson Interferometer for Passive Atmospheric Sounding instrument on board Envisat (MIPAS-E) from July 2002 to March 2004 are used to investigate a possible link between galactic cosmic rays (GCRs) and midaltitude and high-altitude clouds (CI, Occ: 9-18 km, Ext: 12-24 km). The zonally averaged data with $3 \mathrm{~km}$ vertical resolution are averaged over six major Forbush decrease (FD) events and subsequently correlated with the Climax neutron monitor data (CNM). In order to allow for nucleation and growth of ice particles, time lags from 0 to 5 days are applied. We find several weak but statistically significant correlations with an excess of positive cloud-GCR correlations. Introducing a time lag does not enhance the correlations significantly. Subdividing the data in a global grid with $30^{\circ} \times 90^{\circ} \times 3 \mathrm{~km}$ resolution shows higher correlations in some regions. The investigation of the individual FD events yields a heterogeneous picture. Overall, there is a weak tendency toward a positive cloud-CNM correlation. A Kolmogorov-Smirnov test shows that for time shifts from 0 to +5 days a weak GCR-cloud effect is evident in the MIPAS-E measurements. An estimation of the impact of this effect delivers that a $15 \%$ increase in CNM would result in a small decrease in CI (corresponding to an increase in cloud opacity) which is most pronounced at $9 \mathrm{~km}$ altitude $(-9 \%$ to $+0.5 \%)$. For $\log ($ Ext $)$ a decrease of -5 to $0 \%$ is calculated at $12 \mathrm{~km}$ altitude which shifts toward weak positive values at higher altitudes.

Citation: Rohs, S., R. Spang, F. Rohrer, C. Schiller, and H. Vos (2010), A correlation study of high-altitude and midaltitude clouds and galactic cosmic rays by MIPAS-Envisat, J. Geophys. Res., 115, D14212, doi:10.1029/2009JD012608.

\section{Introduction}

[2] In the Fourth Assessment Report of the IPCC (Intergovernmental Panel on Climate Change), the level of scientific understanding of the role of solar modulated galactic cosmic rays (GCRs) in the formation of clouds is classified as very low [Intergovernmental Panel on Climate Change $I P C C, 2007]$. This is the hitherto summary of an ongoing debate which was actuated by Svensmark and Friis Christensen [1997] and which is documented by numerous correlation studies and publications to possible nucleation mechanisms. A comprehensive review is given by Usoskin and Kovaltsov [2008].

\subsection{Correlation Studies}

[3] Using data from the International Satellite Cloud Climatology Project (ISCCP), Svensmark and Friis Christensen [1997] proposed a correlation between GCRs and clouds. This was later confined to a link between GCRs and low marine clouds in midlatitudes [Marsh and Svensmark,

\footnotetext{
${ }^{1}$ ICG-1: Stratosphere, Forschungszentrum Jülich, Jülich, Germany.

${ }^{2}$ ICG-2: Troposphere, Forschungszentrum Jülich, Jülich, Germany.
}

Copyright 2010 by the American Geophysical Union. 0148-0227/10/2009JD012608
2000]. Including data after the year 1994 gives a much weaker correlation which Marsh and Svensmark [2003] explain with a calibration problem in the ISCCP data.

[4] A number of studies put these results into question and warn not to neglect other climate variability factors like ENSO or volcanoes [Farrar, 2000; Sun and Bradley, 2002; Jorgensen and Hansen, 2000]. Pallé [2005] and Usoskin et al. [2006] suggest that the correlation of low clouds with GCR may be an artifact due to low cloud obscuration by higher clouds in the satellite observing perspective. Usoskin et al. conclude that the GCR-cloud relation is an artifact in some regions and a real fact in others. Cloud cover anomalies from 1900 to 1987 over the USA do have a signal at 11 years that is anti-phased with the galactic cosmic ray flux [Udelhofen and Cess, 2001].

[5] The GCR-cloud theory is supported by the fact that the correlation between the ISCCP-D2 low cloud data and modeled cosmic ray ion production has a geographical pattern [Pallé et al., 2004] which shows a latitude dependence with a minimum at the equator [Usoskin et al., 2004]. However, according to Kristjánsson et al. [2004] a direct correlation between solar irradiance variability and low cloud cover delivers better results than between GCR flux and low cloud cover. Voiculescu et al. [2006] find in the ISCCP-D2 data that depending on altitude and geographical position in 
some regions the clouds are mostly affected by UV irradiance and in others by GCRs.

[6] Sloan and Wolfendale [2008] estimate that less than $23 \%$ of the 11 year cycle change in globally averaged cloud cover is due to the change in the rate of ionization from cosmic rays.

[7] The results specified above were obtained from analyses of decadal changes of GCRs and cloud cover. This comprises the disadvantage that they could be interfered by other climate variability like ENSO or volcanoes. This is different if the short-term variability of GCR flux is investigated.

[8] An abrupt decrease in cosmic ray intensity followed by a slow recovery typically lasting for several days is called a Forbush decrease (FD) event. It is caused by passage of an interplanetary transient (usually a shock) typically driven by a strong eruptive solar coronal mass ejection which temporarily sweeps out cosmic rays from the vicinity of Earth. FD events are often accompanied by solar energetic particle (SEP) events. In this case the ionization decrease from the GCR decline is superposed by an ionization increase from solar particles. FD events occur several times per year, depending on solar cycle. Thus, they have no significant effect on climate but provide an opportunity to study the influence of the solar variability without superposition from other climatic dynamics. Several empirical associations have been reported between GCR and clouds during FD events.

[9] Based on the values of total cloud cover from actinometric observatories of the U.S.S.R. Pudovkin and Veretenenko [1995] found a reduction of the high-level cirrus cloud cover associated with Forbush decreases in the latitude range from 60 to $64^{\circ} \mathrm{N}$. Analysis of the ISCCP-D1 data [Todd and Kniveton, 2001, 2004] indicate a reduction of high level clouds - cirrus clouds and polar stratospheric clouds (PSCs) - of $12 \%$ over the Antarctic during the austral winter after FD events. Additionally, the data exhibit statistically significant reductions of cloud cover in regions with relatively high cloud cover at high and equatorial latitudes [Kniveton, 2004]. Also, the diffuse radiation changes from surface radiation measurements across the UK correlate statistically significant with changes in GCR. During FD events, simultaneous decreases occur in the diffuse fraction [Harrison and Stephenson, 2006]. In a recent study which uses data from the Special Sensor Microwave/Imager (SSM/I), Moderate-resolution Imaging Spectroradiometer (MODIS) and ISCCP, Svensmark et al. [2009] report that the fraction of low clouds and the low cloud liquid water content decreases after strong FD events. Additional observations by the aerosol robotic network AERONET measure decreases in the relative abundance of fine aerosol particles. At contrary, by investigating more and hence, also weaker FD events, no systematic correlation was found between cloud parameters from the MODIS instrument and variations in GCR flux in Southern Hemisphere ocean regions [Kristjánsson et al., 2008].

[10] In contrast to the ISCCP-GCR correlations, the shortterm variability of GCR flux also shows correlation with high cloud cover. However, this is confined to isolated regions and short data sets.

\subsection{Nucleation Theory}

[11] GCRs are the main source for ionization in the stratosphere and troposphere. The flux of GCRs is modu- lated by the solar wind (low solar activity corresponding to high GCR flux).

[12] To explain the GCR-cloud cover correlation, two microphysical processes are under consideration: The ionaerosol "near-cloud" theory from Tinsley [Tinsley, 2000; Tinsley and Yu, 2004; Tinsley et al., 2007], also called "electroscavenging," is very complex and will only briefly be mentioned here. According to this theory, changes in GCR alter the space charge near the edges of clouds. Through this space charge highly charged aerosols are produced which can be scavenged by droplets. If the scavenged particle is an ice-forming nucleus supercooled droplets will freeze. This can entail enhanced convection and storminess.

[13] The second microphysical process, the ion-aerosol "clear sky" theory will be explicated in more detail, because it might better apply to the region of our correlation study, the upper troposphere (UT). The first step of the ion-aerosol "clear sky" theory is the ion-induced nucleation (IIN) [Yu and Turco, 2000, 2001; Froyd and Lovejoy, 2003a, 2003b] according to which the GCR ionization affects the production of aerosols. In the lower troposphere, an increase in GCR would lead to an increase in aerosol which might grow to cloud condensation nuclei (CCN) [Yu and Turco, 2001; Yu, 2002]. This causes a decrease in droplet size and hence an increase of cloud reflectivity and cloud lifetime. In recent years, enormous progress has been made in the ion-induced aerosol nucleation theory [Enghoff and Svensmark, 2008; Kazil et al., 2008].

[14] The effect of IIN should be greatest in the lower atmosphere where enough condensable gases $\left(\mathrm{H}_{2} \mathrm{SO}_{4}\right)$ are available and the nucleation rate of ultrafine aerosols is limited because of the ion concentration. Studies from a numeric nucleation model [Lovejoy et al., 2004; Curtius et al., 2006] indicate that IIN also explains nucleation events in the remote middle troposphere.

[15] For the upper troposphere (UT) - hence, the region of our correlation study - the prediction is less clear. $Y u$ [2002] calculates that in the UT an increase in cosmic ray fluxes leads to a decrease in aerosol production. This is explained by a reduced lifetime of ion clusters due to the increase in ionization rate. By applying the Lovejoy et al. [2004] model to the conditions studied by $Y u$ [2002], Kazil and Lovejoy [2004] find a positive correlation of aerosol formation and ionization throughout the troposphere. However, depending on the conditions, either a negative or positive correlation was calculated. $Y u$ et al. [2008] calculate with a global chemical transport model (GEOS-Chem) that ion-mediated aerosol nucleation is significant in the tropical UT, the entire middle latitude troposphere, and over Antarctica.

[16] Several observational studies have provided evidence for the existence of IIN. Large positive cluster ions in the UT have been detected by Eichkorn et al. [2002]. Lee et al. [2003] measured high concentrations of ultrafine particles in the upper troposphere/lower stratosphere (UT/LS) which were consistent with a simulation by a numerical model of IIN. Svensmark et al. [2007] found in laboratory experiments that ions are active in generating thermodynamically stable cluster.

[17] In a detailed study including laboratory measurements, atmospheric measurements, and model simulations, Arnold [2008] shows that both IIN and homogeneous 

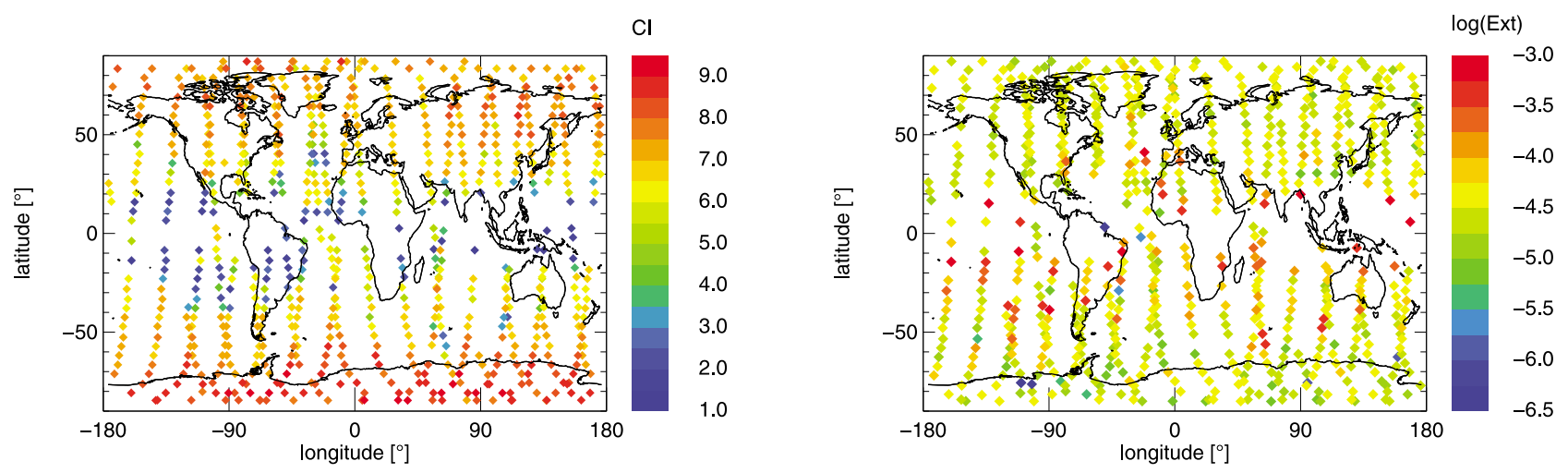

Figure 1. Geo-located maps of (left) CI and (right) $\log$ (Ext) for one day of measurement (2003-11-15, $15 \mathrm{~km}$ altitude).

nucleation are frequent and efficient processes in the cold UT. He concludes that cosmic ray mediated formation of CCN sized particles should at least occasionally be operative in the UT. However, the growth of ultrafine aerosol particles to CCN size is the critical step of the ion-aerosol "clear sky" mechanism. In a recent model study, Pierce and Adams [2009] calculate that changes in CCN from changes in GCRs are too small to account for the observed changes in cloud properties.

[18] The main part of the previous studies which have found a GCR-cloud link have used the ISCCP-data set. Due to the nadir view of these satellites, they tend to overlook optically thin cirrus clouds at high altitudes or polar stratospheric clouds (PSC). We think that it is worth looking for a possible connection between GCR and mid and high altitude clouds (cirrus + PSC) by using data from the limb viewing satellite instrument MIPAS.

[19] Infrared measurements in the limb are extremely sensitive to detect optically thin clouds like already presented for various instruments [e.g., Mergenthaler et al., 1999; Hervig and McHugh, 1999; Massie et al., 2002; Spang et al., 2002]. The long limb path through the atmosphere (typically $200-300 \mathrm{~km}$ in the tangent height layer) and consequently long path through a cloud structure allows the detection of optically thin clouds like subvisible cirrus (SVC), ultrathin tropical tropopause cirrus (UTCC), or various PSC types. This and the typically good vertical resolution are significant advantages of limb sounders compared to the operational nadir looking instruments used in the ISCCP data. However, these advantages are partly compensated by the only poor and moderate horizontal sampling and coverage of limb occultation (e.g., HALOE and SAGE instruments) and limb emission measurements (e.g., MIPAS, for details see Section 2.1), respectively.

\section{Data and Methods}

\subsection{MIPAS-E}

[20] In this study we use the extinction data (Ext), the occurrence frequency (Occ) as well as the cloud index data (CI) from the Michelson Interferometer for Passive Atmospheric Sounding (MIPAS). MIPAS was launched on the Envisat satellite in March 2002 and is providing limb sounding spectra of atmospheric infrared emission between $685 \mathrm{~cm}^{-1}(14.60 \mu \mathrm{m})$ and $2410 \mathrm{~cm}^{-1}(4.15 \mu \mathrm{m})$ at a spectral unapodized resolution of $0.025 \mathrm{~cm}^{-1}$ [Fischer and Oelhaf, 1996; Fischer et al., 2008]. The emission measurements and the sun-synchronized orbit give the opportunity for day and night measurements from pole to pole of around 1200 limb sequences per day. As a limb-viewing satellite instrument MIPAS offers a good vertical resolution relative to nadir instruments. Vertical sampling and resolution of the instrument is in the order of $3 \mathrm{~km}$. The horizontal sampling is relatively sparse compared to nadir instruments and is in the order of $600 \mathrm{~km}$. The horizontal measurement distribution is illustrated in Figure 1 by an example for the so-called cloud index and extinction data for one day of measurements at $15 \mathrm{~km}$ altitude (for details see next Sections). Measurement gaps near the equator are caused by high optically thick clouds (here at around $18 \mathrm{~km}$ ) which obscure the view to lower altitudes. Small CI respectively large extinction values represent the presence of clouds.

[21] At the end of March 2004, MIPAS operation was stopped due to problems with the mirror drive of the interferometer and later resumed in the so-called reduced resolution mode. In this study we only use the data from July 2002 to March 2004 measured with full spectral resolution. The total amount of data in this period represents ca. 550000 profiles.

[22] During measurements in the full resolution mode MIPAS used a nominal vertical sampling grid of $3 \mathrm{~km}$ at fixed altitudes of $6,9,12, \ldots, 30 \mathrm{~km}$. The post flight altitude data vary only slightly with time and latitude from the nominal heights $( \pm 1 \mathrm{~km})$. In the following statistical analyses the data are binned in the vertical by $3 \mathrm{~km}$ intervals which are centered at the nominal altitudes. By this approach the altitude of the effective bin centers are quite well represented by the nominal altitude grid. In the most disadvantageous case they vary by $\pm 1.3 \mathrm{~km}$.

2.1.1. Cloud Index and Cloud Occurrence Frequency

[23] The high spectral resolution provides the separation between the line emission of the atmospheric trace gases and the more continuum-like emission of clouds and aerosols. Spang et al. [2002, 2004, 2005a] have developed a very reliable method for the detection of clouds by using the ratio of mean radiances from two different wavelength regions. The first micro-window (MW1) is dominated by trace gases, the second micro-window (MW2) is mainly dominated by aerosols and clouds. The cloud indices CI-A (MW1 788.2$796.25 \mathrm{~cm}^{-1}$, MW2 832.3-834.4 $\mathrm{cm}^{-1}$ ) and CI-D (MW1 


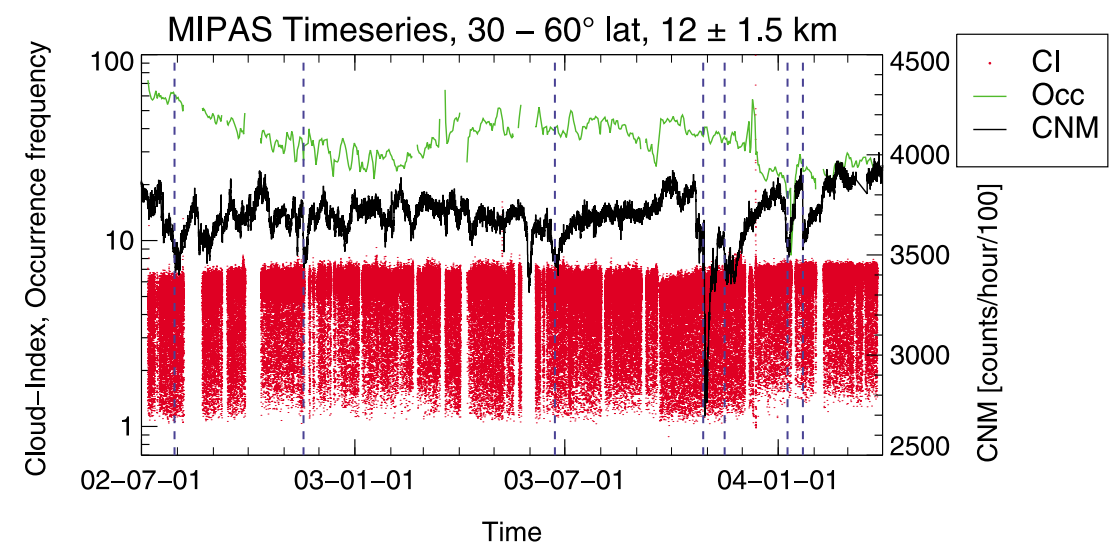

Figure 2. MIPAS-E cloud index (CI) and cloud occurrence frequency Occ (\%) time series between 30 and $60^{\circ} \mathrm{N}$ at an altitude range from 11 to $13 \mathrm{~km}$ and Neutron Count data from July 2002 until March 2004; vertical lines indicate the included FD events.

1929.0-1935.0 $\mathrm{cm}^{-1}$, MW2 1973.0-1983.0 $\mathrm{cm}^{-1}$ ) [Spang et al., 2004] were chosen for the investigation of the GCRcloud link and were calculated for the first time period of MIPAS-E measurements (July 2002-March 2004). The correlation analysis of both CI-A and CI-D with GCR gave very similar results. Due to a better signal-to-noise ratio CI-A was chosen for the further analysis. The abbreviation CI stands for CI-A hereinafter.

[24] CI is a measure for cloud transparency - it decreases rapidly from one altitude step to the next from background values of around $6-10$ to $1-4$ if a cloud is in the field-ofview. In this study $\mathrm{CI}<1.5$ is chosen as a threshold for optically thick conditions. From a profile all tangent heights below the highest tangent height with $\mathrm{CI}<1.5$ are excluded from the analysis. CI $>4.5$ does indicate cloud-free conditions. This method has been used already for intensive analyses for detection, classification and validation of PSC occurrence in both polar vortices in the MIPAS data [e.g., Spang et al., 2005a, 2005b; Höpfner et al., 2006a, 2006b] as well as for the detection of cirrus clouds [Spang et al., 2004; Greenhough et al., 2005]. The results show the reliability of the data set and the applied analysis methods.

[25] We define the cloud occurrence frequency [Occ] as the number of cloud events with $\mathrm{CI}<4.5$ divided by the total number of CI observations in a time-window of one day and an altitude and latitude-longitude grid box.

[26] Figure 2 shows as an example the MIPAS-E cloud index and occurrence frequency time series between 30 and $60^{\circ} \mathrm{N}$ at an altitude interval at $12 \pm 1.5 \mathrm{~km}$. The CI data scatter between 1 (thick cirrus) and 10 (cloud free), the Occ data vary between 7 and $80 \%$.

\subsubsection{Aerosol Extinction Coefficients}

[27] To retrieve the aerosol extinction coefficients the rapid forward model JURASSIC, developed at the Research Centre Jülich [Hoffmann, 2006], and the standard optimal estimation approach [e.g., Rodgers, 2000] have been applied to the MIPAS level $1 \mathrm{~b}$ radiance data like described by Hoffmann et al. [2008] for the CFC-11 retrieval of MIPAS.

[28] The micro-window selection for the retrieval had to be optimized in respect to changes in the continuum part of the spectra due to aerosol variation and interfering radiances by other trace gases. The Shannon information content was chosen as figure of merit to be optimized for the spectral window selection under consideration of MIPAS instrument errors (e.g., noise and uncertainties in radiometric calibration as well as forward model parameter errors like uncertainties in pressure, temperature, or interfering species) (for details, see Hoffmann et al. [2008]). The spectral window 832.3$834.4 \mathrm{~cm}^{-1}$ was identified as being one of the best suited for extinction retrievals and was selected for the processing of all MIPAS measurements in the period July 2002 to March 2004. Information on temperature, pressure, and contaminant species were used from the ESA operational level 2 products. To identify and remove extremely cloud contaminated data which are not retrievable with the recent forward model we applied a threshold value of $\mathrm{CI}=2$ as cloud detection limit to the MIPAS level $1 \mathrm{~b}$ data. The retrieval is stopped at the highest tangent altitude of observation where CI is below this threshold. In consequence the retrieved extinction data are a combination of two emitters. One fraction is caused by the background aerosol which is recently generally quite low in the upper troposphere and lower stratosphere due to the low background aerosol load and one fraction by any additional emissions from optically thin or even subvisible cirrus clouds. An enhanced extinction corresponds to enhanced aerosol and/ or cloud load.

[29] Noise and calibration errors of the level $1 \mathrm{~b}$ radiances are the dominant retrieval errors. The extinction error of single profile value is in the order of $10^{-5} \mathrm{~km}^{-1}$ around the tropopause and $10^{-6} \mathrm{~km}^{-1}$ at the maximum retrieval height of $40 \mathrm{~km}$ [Spang et al., 2007]. For the correlation analysis with the neutron monitor data (CNM) we use the logarithm of the extinction data which is abbreviated as $\log (\mathrm{Ext})$ in the following. Figure 3 shows as an example the MIPAS-E aerosol extinction time series between 30 and $60^{\circ} \mathrm{N}$ at an altitude interval of $12 \pm 1.5 \mathrm{~km}$. The $\log (\mathrm{Ext})$ data scatter in a range from -3 to -7 with the bulk of the data between -4 and -5 . Values less than -5 are in the range of the retrieval noise.

\subsection{Neutron Counts}

[30] The neutron monitor data (CNM) were taken from the Climax station (Climax, Colorado, $39.4^{\circ} \mathrm{N}, 253.8^{\circ} \mathrm{E}$ ). Additionally, the neutron monitor data from the HALEAKALA station (HNM, Haleakala, Hawaii, $20.7^{\circ} \mathrm{N}, 203.7^{\circ} \mathrm{E}$ ) were 


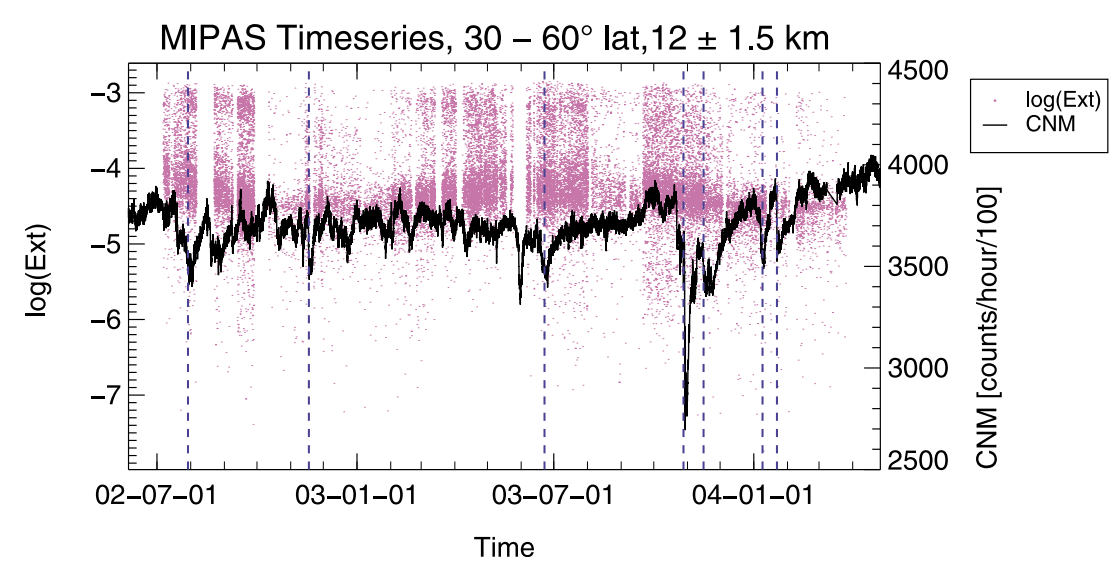

Figure 3. MIPAS-E aerosol extinction time series between 30 and $60^{\circ} \mathrm{N}$ at an altitude range from 11 to $13 \mathrm{~km}$ and Neutron Count data from July 2002 until March 2004; vertical lines indicate the included FD events.

used. The results showed no significant difference and are not presented in detail.

[31] A Forbush decrease event was defined as a $>5 \%$ suppression of the hourly count rate of the Climax neutron monitor with respect to the 100-day running mean. During the analyzed time period 6 FD events occurred (Table 1 and vertical blue lines in Figures 2 and 3). In addition, after the solar flare on 2003-10-28 (Halloween-Flare) an extreme energetic solar particle (SP) event passed the earth after less than one day followed by a depletion of GCRs of more than 20\% [Flückiger and Bütikofer, 2004]. Due to a possible superposition from the SP event which should effect mainly the polar stratosphere [Bazilevskaya et al., 2008], the HalloweenFlare is omitted from the analysis.

\subsection{Statistical Methods}

\subsubsection{Selection of the Geographical and Temporal Resolution}

[32] For the correlation analysis between two data sets which possess also internal variation, the choice of the averaging method of the data can be crucial. On one hand, averaging might help to detect a weak correlation by flattening the internal variability; on the other hand, one might loose sharpness by mixing different processes.

[33] The vertical resolution of $3 \mathrm{~km}(9-18 \mathrm{~km}$ altitude for CI and Occ and 12-24 km for $\log ($ Ext)) is given by the MIPAS vertical grid. However, the geographical and temporal resolution depends on the amount of available data and the natural spatiotemporal variability and is, somehow, arbitrary.

[34] We chose a latitudinal resolution of 30 degrees. Due to this averaging over the whole latitude bands, meteorological variability should be evened out to a large extent. Only the analysis of the polar latitudes might still be highly influenced by meteorological variability. Furthermore, we partition the latitude bands in 4 longitudinal sections of $90^{\circ}$. A finer splitting results in too low numbers of data points.

[35] For investigating the cloud-GCR link, we correlate the whole time series of the MIPAS cloud parameters with the CNM data. However, the focus lies on a superposed epoch (SPE) analysis of the 21 day periods around the FD events. Here, we calculate the average values of the $6 \mathrm{FD}$ events for the MIPAS cloud parameters and the CNM data. There are not always data in each of the $6 \mathrm{~h}$ cloud parameter bins of the 6 single FD events. So sometimes less then 6 cloud parameter and CNM data points are averaged. This enhances the standard deviation of the averaged data point and thereby, the effect is reflected in the error analysis. This reduction mainly occurs at low altitudes, because of obscuration through high altitude clouds.

[36] In addition, we analyze the time periods from -10 to +10 days around each individual FD event.

[37] For the right choice of the temporal resolution we performed a variance analysis analog to Rohrer and Berresheim [2006]. The data were binned into series of time intervals ranging from $1 \mathrm{~h}$ to the whole time range of 21 days. As an example, in Figure 4 the results of the variance analysis of the hourly mean CI and CNM data of the combined $6 \mathrm{FD}$ events between 0 and $30^{\circ} \mathrm{S}$ at an altitude range from $9 \pm 1.5 \mathrm{~km}$ are shown.

[38] The main part of the variance of the CNM data occurs at time scales of more than one day, but a significant part also proceeds at a time scale of some hours. In the selected example, the variance of CI reaches $100 \%$ in less than half a day. So, by correlating daily mean data, we would loose this time range with a possible relation between the two data sets. Therefore, we chose $6 \mathrm{~h}$ averages of the CI and log (Ext) data for the correlation analysis. The Occ data exist as daily means which might be a disadvantage for the correlation study. Examples for 20 day periods of the 6 hourly mean CI, $\log (\mathrm{Ext})$ and daily mean Occ data of the individual

Table 1. Date and Magnitude of the Six Investigated Forbush Decrease Events ${ }^{\mathrm{a}}$

\begin{tabular}{cc}
\hline Date & Magnitude (\%) \\
\hline $2002-07-29$ & -7.7 \\
$2002-11-17$ & -7.1 \\
$2003-06-22$ & -6.8 \\
$2003-11-15$ & -7.8 \\
$2004-01-09$ & -6.4 \\
$2004-01-22$ & -6.6 \\
\hline
\end{tabular}

${ }^{a}$ Dates given as yyyy-mm-dd. The magnitude is defined as the decrease of the hourly count rate of the Climax neutron monitor with respect to the 100 -day running mean. 


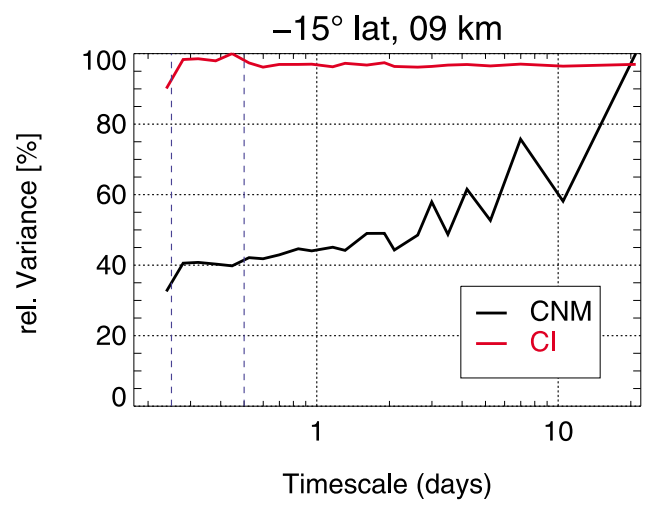

Figure 4. Variance analysis of the hourly mean CI and CNM data of the combined 6 FD events between 0 and $30^{\circ} \mathrm{S}$ at an altitude range from $9 \pm 1.5 \mathrm{~km}$; the blue line indicates time scales of 6 and $12 \mathrm{~h}$; maximal variance of CNM: 8858 (counts/hour) ${ }^{2}$, maximal variance of CI: 0.26 .

FD events and the averages over the $6 \mathrm{FD}$ events for $\mathrm{CI}$, log (Ext), Occ and CNM are given in Figure S1 the in the auxiliary material. ${ }^{1}$

\subsubsection{Selection of Time Lags}

[39] In order to allow for nucleation and growth of ice particles but also to identify statistical artifacts, these studies were performed with time lags ranging from -5 to 5 days $(-5,-3,-1,0,1,3,5$ days). By shifting the 20 day-periods of the 6 FD events, we find a strong autocorrelation of CNM with a maximum at -20 days. This is caused by the autocorrelation due to the solar rotation period of 27 days and by the short span of time between the Halloween Flare and the FD event from 2003 to 11-15 (Figure 2). In the same manner, a strong negative autocorrelation occurs after a time shift of +15 days. If, without time shift, there is a high correlation between a cloud parameter and CNM, this inevitably causes also a high correlation at -20 days and a high anticorrelation at +15 days. To avoid problems resulting from this autocorrelation, a maximum time shift of \pm 5 days (less than 15/2) was chosen.

\subsubsection{Calculation of the Significance}

[40] The assessment of the significance whether a correlation between a MIPAS cloud parameter and CNM is different from $r=0$ turned out to be very complex. We applied a series of independent statistical tests (e.g., standard formula, bootstrap, box plot, method of Ebisuzaki [1997]). The standard formulas which are based on the precondition that the data points are independent and Gaussian distributed overestimate the significance.

[41] Finally the non-parametric method according to Ebisuzaki [1997] which takes the serial correlation between the data points into account has been proven to be best suited and was applied here. This method consists of a FFT transformation of the original data series, randomization of the phase while keeping the amplitude, and an inverse FFT transformation. Then, the randomized data series is correlated with the original second data series (with the correlation coefficient $r_{\text {rand }}$ ). This procedure is conducted $\mathrm{N}$ times (we chose $\mathrm{N}=2000$ ). The $\mathrm{p}$-value is the fraction of cases

\footnotetext{
${ }^{1}$ Auxiliary materials are available in the HTML. doi:10.1029/ 2009JD012608.
}

with $\left|r_{\text {rand }}\right|>|r|$ (correlation coefficient of the original correlation without randomization). Then, the whole procedure is repeated for the second data series (while keeping the first data series unmodified) and the lower significance is chosen as the estimated value. We regard a significance level $>95 \%$ ( $p$-value $<0.05)$ as significant and a significance level between 90 and $95 \%(0.05<\mathrm{p}$-value $<0.10)$ as marginally significant. According to Usoskin et al. [2006] this method may underestimate the significance of the correlation if the power spectrum of the original series is dominated by a single strong peak. Overall, the method of Ebisuzaki [1997] yields significance levels which depend not only on the number of data points but also on the amount of autocorrelation.

\subsubsection{Kolmogorov-Smirnov Test}

[42] In addition to the estimation of the significance of single data points, it is of interest, whether the correlation coefficients of all latitude-longitude grids as a whole differ from a random distribution. For this purpose we use the Kolmogorov-Smirnov test. It has the advantage of being nonparametric and distribution free. In computing the maximum difference between the experimental and the theoretical (random) distribution functions and comparing it with the tabulated critical D-value for that sample, the KolmogorovSmirnov test determines if two data sets differ significantly [Brandt, 1999; Sachs and Hedderich, 2006].

\section{Results}

[43] We correlate the cloud index (CI), the logarithm of the extinction $(\log (E x t))$ and the cloud occurrence frequency (Occ) data with the neutron count data from the Climax station (CNM). With these data we have a very sensitive tool for the investigation of high clouds. While the cloud index and the occurrence frequency are more directly related to cloud effects, the retrieved extinctions in the UT/LS region are also sensitive to the cosmic ray induced aerosol effects as precursors for clouds. The CI and Occ data are calculated for an altitude range from 9 to $18 \mathrm{~km}$ altitude except for the midlatitudes and polar summer regions at 15 and $18 \mathrm{~km}$ altitude where no clouds exist. The $\log (\mathrm{Ext})$ data are calculated for an altitude range from $12-24 \mathrm{~km}$ altitude.

[44] We selected three different time windows: (1) the whole time series, (2) the combined 20-day periods around

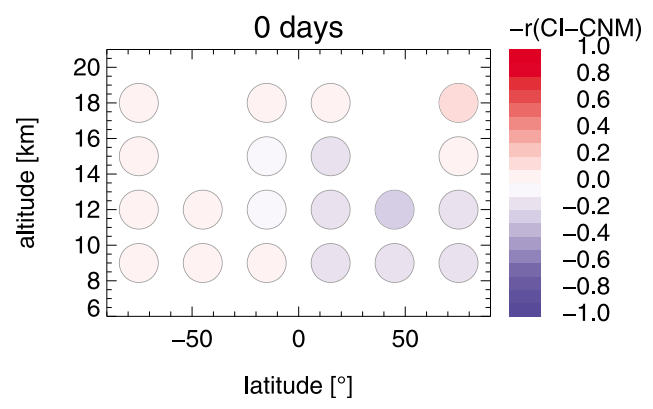

Figure 5. Latitude-altitude distribution of the correlation coefficient $r$ between $\mathrm{CI}$ and CNM for the whole time series without time shift: all correlations have $\mathrm{p}$-values $>0.1$; for better comparison with $\log (\mathrm{Ext})$ and Occ the negative of the correlation is plotted. 

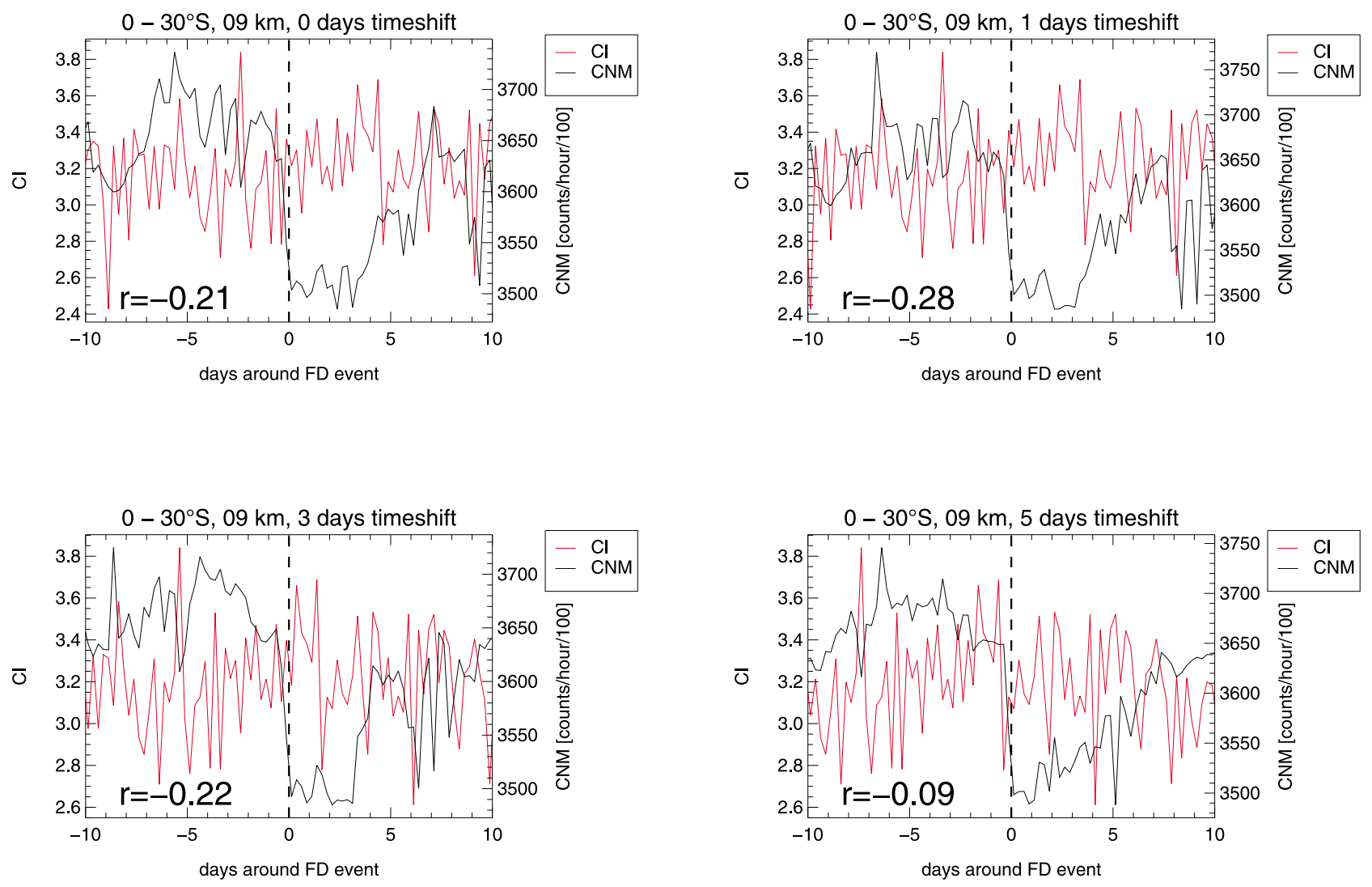

Figure 6. Twenty day periods of the 6 hourly mean CI and CNM data averaged over the 6 FD events: 0 $30^{\circ} \mathrm{S}, 9 \pm 1.5 \mathrm{~km}$ for $0,1,3,5$ days time shift.

the 6 Forbush decrease events, and (3) the individual 20-day periods around the Forbush decrease events.

\subsection{CI-CNM Correlation}

[45] The cloud index (CI) measures the cloud transparency, so a mean CI can be interpreted as a measure for the mean optical thickness of the cloud cover. We calculated the correlation coefficients between the hourly mean CI and CNM data from the whole time series of 1.5 years. The calculated values are summarized in Figure 5 for all investigated latitude and altitude intervals. Since a low CI belongs to an optically thick cloud cover, a negative CI-CNM correlation coefficient corresponds to a positive cloud-CNM correlation coefficient and vice versa. For a better comparison with the $\log (\mathrm{Ext})-\mathrm{CNM}$ and Occ-CNM correlations, we have plotted the inverse of the correlation.

[46] Blue points indicate positive correlations between CI and $\mathrm{CNM}$ and, hence, negative correlations between the mean optically thickness of the cloud cover and CNM. Vice versa, red points indicate positive correlations between the mean optical thickness of the cloud cover and CNM. For the whole time series, we did not find any significant correlation between CI and CNM. Actually, this is hardly surprising, because - if any - we expect only a small effect from GCRs on mid and high clouds. So, this effect can easily be superimposed by normal atmospheric variability.

[47] More promising is the SPE analysis of the timeintervals of the $6 \mathrm{FD}$ events. In Figure 6 the CI and CNM data, averaged over the $6 \mathrm{FD}$ events from 10 days prior to the FD event to 10 days past the event are exemplified for $0-30^{\circ} \mathrm{S}, 9 \pm 1.5 \mathrm{~km}$. Note that the CNM curve looks different in each plot, because some of the $6 \mathrm{~h}$ bins of the 6 single FD events do not contain CI data (see Section 2.3.1); an effect which mainly affects low altitudes.

[48] In the example which is shown in Figure 6, the negative correlation coefficient increases from $\mathrm{r}=-0.22$ at a time shift of 0 days to $r=-0.28$ at a time shift of 1 day and decreases afterwards again. Albeit a relation between the CI and CNM data is hardly visible, the correlations from 0 to 3 days time shift are nominally significant. However, due to the low values of $r$, this relation can only explain $4.8-7.8 \%$ of the observed variances.

[49] The calculated correlation coefficients for the combined data from the $6 \mathrm{FD}$ events are presented in Figure 7. Grey circles in Figure 7 mark correlation coefficients with $0.05<\mathrm{p}<0.1$ and black circles correlation coefficients with $p<0.05$. The values of $r$ and $p$ with $p<0.1$ are given in Table 2. The ratio of negative to positive CI-CNM correlation areas is 2.7. A negative correlation means that with increasing GCR flux the CI decreases and hence, the mean thickness of the cloud cover increases. In addition to the correlation at $0-30^{\circ} \mathrm{S}, 9 \pm 1.5 \mathrm{~km}$ which is shown in Figure 6 we find a marginally significant correlation at $60-90^{\circ} \mathrm{N}, 15 \pm$ $1.5 \mathrm{~km}$ as well as another significant negative correlation of $\mathrm{r}=-0.29$ at $30-60^{\circ} \mathrm{S}, 12 \pm 1.5 \mathrm{~km}$. The latter correlation becomes more significant at 1 day time shift, where also a 

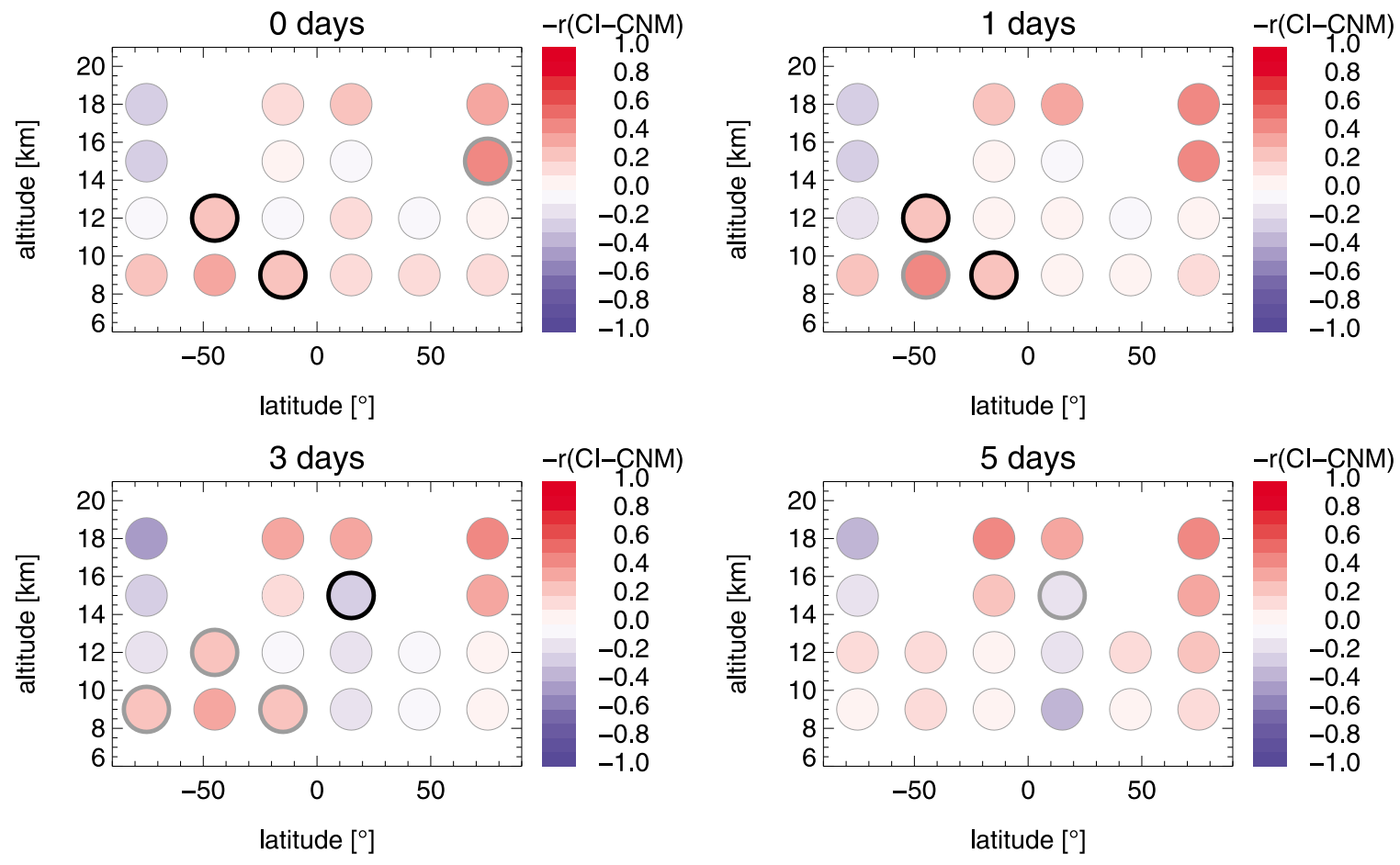

Figure 7. Same as Figure 5 but for the combined 6 FD events at 0,1, 3 and 5 days time shift, gray circles indicate correlations with $0.05<\mathrm{p}<0.1$, black circles indicate correlations with $\mathrm{p}<0.05$; for better comparison with $\log (\mathrm{Ext})$ and Occ the negative values of $\mathrm{r}$ are plotted.

marginally significant negative correlation becomes evident at $30-60^{\circ} \mathrm{S}, 9 \pm 1.5 \mathrm{~km}$. After 3 days, these correlations are weaker again and an additional marginally significant negative correlation at $60-90^{\circ} \mathrm{S}, 9 \pm 1.5 \mathrm{~km}$ as well as a significant positive correlation of $\mathrm{r}=+0.24$ at $0-30^{\circ} \mathrm{N}, 15 \pm$
$1.5 \mathrm{~km}$ emerge. This positive correlation is also seen for a time shift of 5 days. For comparison, the same plot but with HNM data from the HALEAKALA station is shown in the auxiliary material (Figure S2). It exhibits the same features but with slightly more positive cloud-NM correlations.

Table 2. Correlation Coefficients $r$ and $p$-Values of Significant Correlations Between CNM and CI, $\log ($ Ext), and Occ for the Combined Six FD Events ${ }^{\mathrm{a}}$

\begin{tabular}{|c|c|c|c|c|c|c|c|c|}
\hline Time Shift (days) & Latitude $\left({ }^{\circ} \mathrm{N}\right)$ & Altitude $(\mathrm{km})$ & $\mathrm{r}(\mathrm{CI})$ & p-Value (CI) & $r(\log (E x t))$ & p-Value $(\log ($ Ext $))$ & $\mathrm{r}(\mathrm{Occ})$ & $\mathrm{p}$-Value (Occ) \\
\hline 0 & -75 & 12 & - & - & - & - & -0.65 & 0.036 \\
\hline 0 & -45 & 9 & - & - & - & - & 0.55 & 0.032 \\
\hline 0 & -45 & 12 & -0.29 & 0.048 & - & - & 0.61 & 0.032 \\
\hline 0 & -45 & 18 & - & - & -0.41 & 0.052 & - & - \\
\hline 0 & -15 & 9 & -0.22 & 0.036 & - & - & - & - \\
\hline 0 & -15 & 12 & - & - & -0.29 & 0.043 & - & - \\
\hline 0 & 15 & 9 & - & - & - & - & 0.52 & 0.054 \\
\hline 0 & 15 & 12 & - & - & 0.25 & 0.022 & - & - \\
\hline 0 & 75 & 15 & -0.40 & 0.097 & - & - & - & - \\
\hline 1 & -75 & 12 & - & - & - & - & -0.70 & 0.012 \\
\hline 1 & -45 & 9 & -0.40 & 0.068 & - & - & 0.53 & 0.030 \\
\hline 1 & -45 & 12 & -0.28 & 0.034 & - & - & 0.62 & 0.034 \\
\hline 1 & -45 & 18 & - & - & -0.46 & 0.054 & - & - \\
\hline 1 & -15 & 9 & -0.28 & 0.005 & & & 0.59 & 0.058 \\
\hline 1 & -15 & 12 & - & - & -0.26 & 0.044 & - & - \\
\hline 1 & -15 & 18 & - & - & - & - & -0.55 & 0.072 \\
\hline 1 & 15 & 12 & - & - & 0.22 & 0.083 & - & - \\
\hline 1 & 45 & 12 & - & - & - & - & -0.29 & 0.090 \\
\hline 3 & -75 & 9 & -0.20 & 0.083 & - & - & - & - \\
\hline 3 & -45 & 12 & -0.22 & 0.068 & - & - & - & - \\
\hline 3 & -15 & 9 & -0.22 & 0.050 & - & - & 0.63 & 0.057 \\
\hline 3 & 15 & 15 & 0.24 & 0.031 & -0.25 & 0.072 & -0.45 & 0.075 \\
\hline 3 & 75 & 12 & - & - & -0.20 & 0.084 & - & - \\
\hline 5 & 15 & 12 & - & - & - & - & -0.51 & 0.045 \\
\hline 5 & 15 & 15 & 0.17 & 0.065 & - & - & -0.47 & 0.055 \\
\hline
\end{tabular}

${ }^{\mathrm{a}}$ The correlations are given for time shifts of $0,1,3$, and 5 days. 

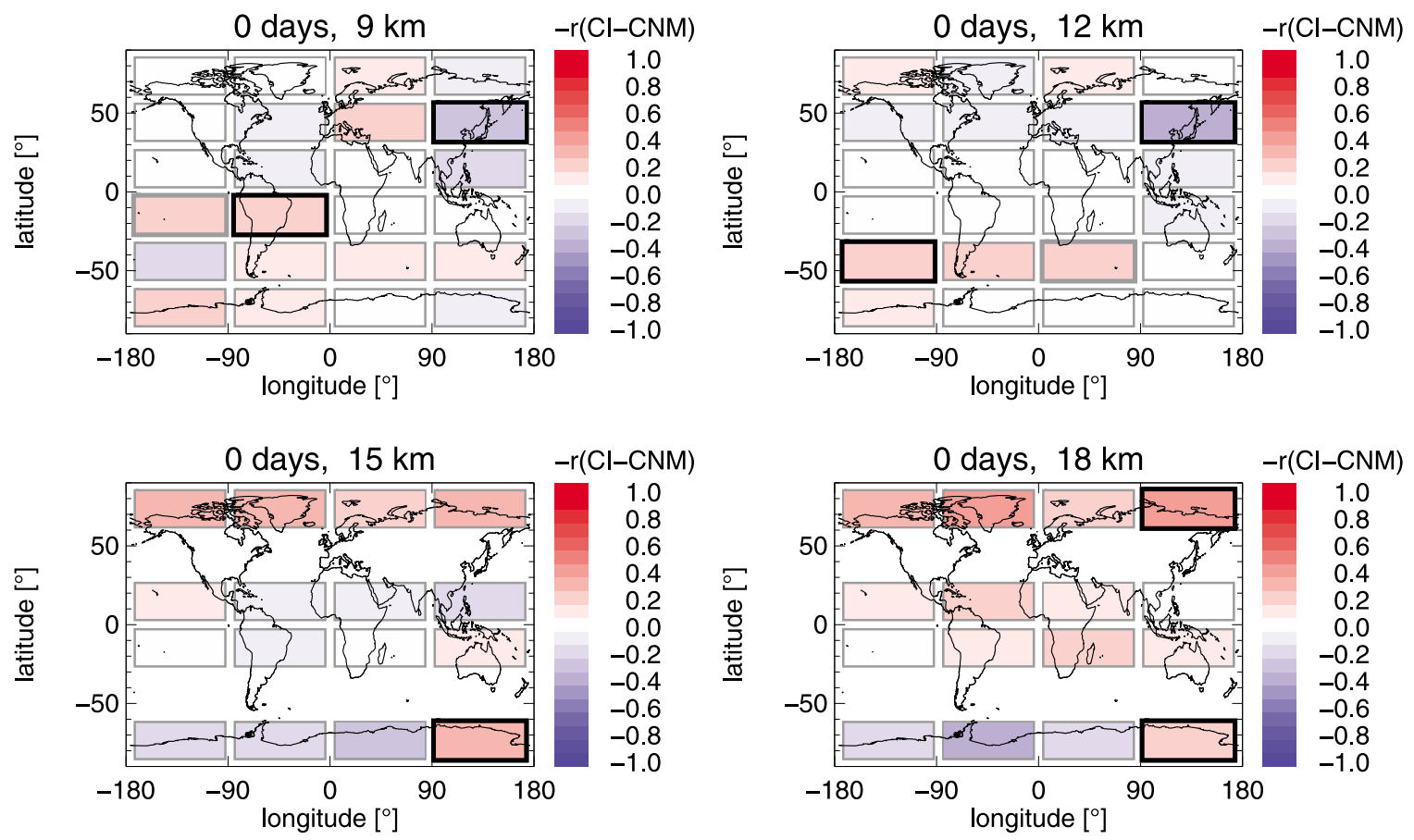

Figure 8. Latitude-longitude distribution of the correlation coefficients $r$ between CI and CNM for the combined 6 FD event at different altitudes (without time shift); for better comparison with $\log (\mathrm{Ext})$ and Occ the negative values of $\mathrm{r}$ are plotted.

[50] For a more detailed view we partitioned the latitude bands in 4 longitudinal sections of $90^{\circ}$ (Figure 8). As expected from the analysis of the whole latitude bands, positive correlations between CNM and cloud cover prevail - in term of spatial areas by a factor of 2.4. For given latitude and altitude in many cases, one sign of the correlation coefficient dominates for the whole longitude range, generating a somewhat striped appearance of the distribution plot. We do not want to interpret these correlation coefficients in too much detail, because, in comparison with the correlation analyses of the whole latitude band, the number of data points which are used for the correlation is reduced from ca. 7000 to 1700 for each individual latitude-longitudealtitude grid.

[51] We also investigated the 6 individual FD events for significant correlations. There is no preferred direction of the correlations. Without time shift, at the FD event of 200306-22 (Figure 9, left) most correlations are slightly positive. At contrary, the FD events of 2003-11-15 (Figure 9, right) and 2004-01-09 (not shown) possess mainly negative correlations and the other FD events show a mixture of positive and negative correlations.

[52] Particularly at high latitudes it is necessary to look at the individual FD events, because 2 of the FD events occurred during boreal summer and 4 during boreal winter. So, we should distinguish between correlations with and without possible formation of PSCs.

[53] From the $4 \mathrm{FD}$ events in boreal winter at $60-90^{\circ} \mathrm{N}$ for all altitudes 13 correlations between $\mathrm{CI}$ and $\mathrm{CNM}$ are negative, 1 neutral and 2 positive. Contrariwise, from the $2 \mathrm{FD}$ events at southern winter, no negative, 4 neutral and 4 positive correlations result. At northern summer $\left(60-90^{\circ} \mathrm{S}, 9\right.$ and
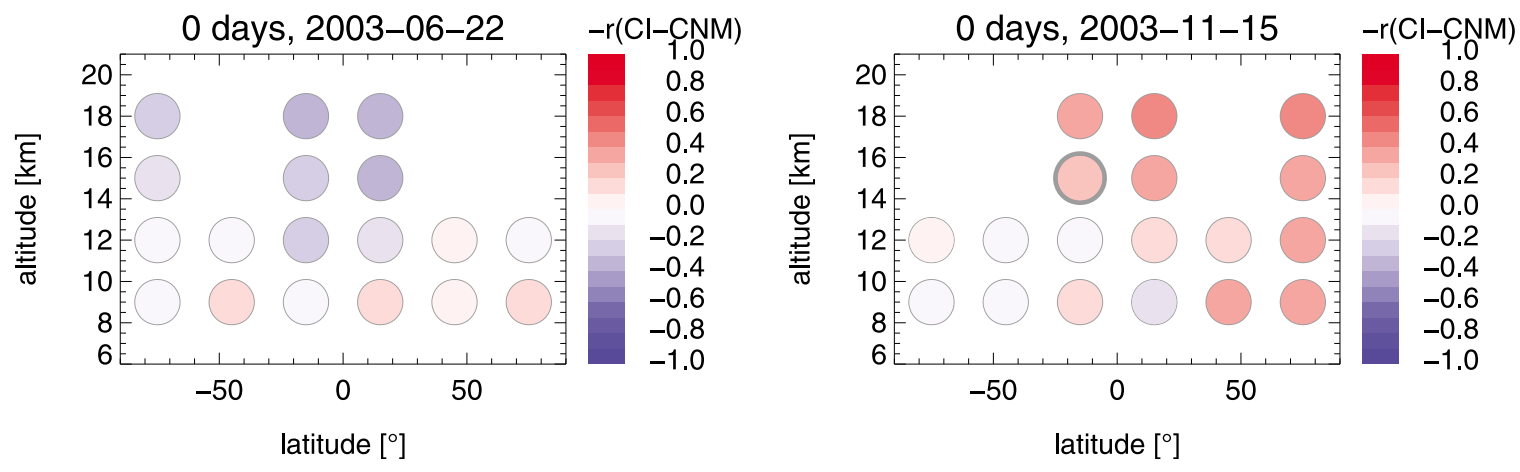

Figure 9. Same as Figure 5 but for individual FD events: (left) 2003-06-22 and (right) 2003-11-15; for better comparison with $\log (\mathrm{Ext})$ and Occ the negative values of $\mathrm{r}$ are plotted. 


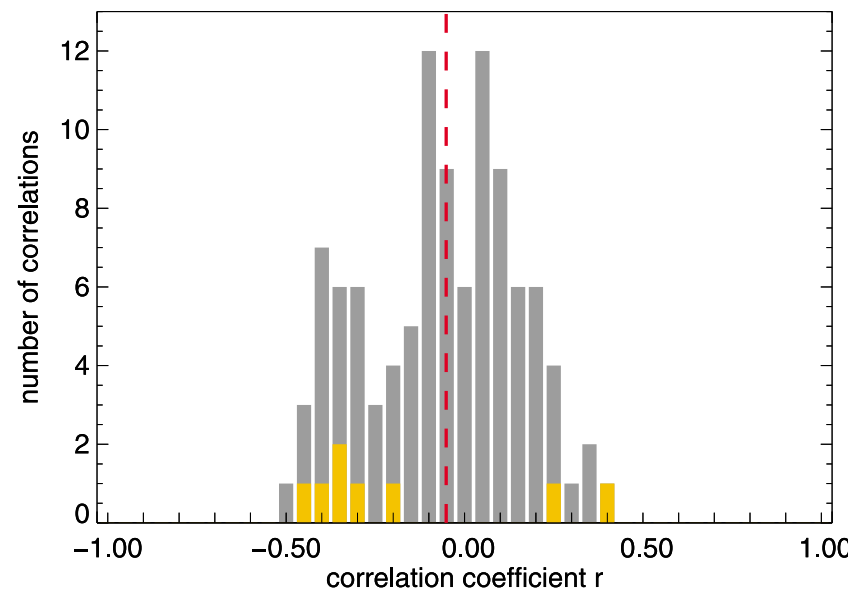

Figure 10. Histogram of correlation coefficients $r$ between CI and CNM for all 6 FD events (all latitudes and altitudes, 0 days time shift); orange color indicates $\mathrm{p}<0.1$; the vertical dashed line indicates the mean of $r$.

$12 \mathrm{~km}$ altitude) where were only 4 correlations (2 events, 2 altitudes) which is insufficient for this analysis, at southern summer 5 correlations are negative, 2 neutral and 0 positive. Hence, at high latitudes in northern winter and in southern summer the negative correlations prevail, so no clear conclusion can be made from this result.

[54] Figure 10 shows the histogram of the correlation coefficients, that is the frequency distribution of all individual events, all latitudes and all altitudes. Correlation coefficients with $\mathrm{p}<0.1$ are marked in orange. The correlation coefficients are distributed around $r=0$. So, few correlations at the edges might fall outside the $95 \%$ significance boundary by chance and not by an existing causal dependency. The histogram seems to feature a bimodal distribution. This is mainly caused by the FD events of 2003-11-15 and 2004-01-09. Therefore, more FD events are needed to ascertain, whether the distribution remains bimodal or whether this was just an artifact.

[55] For time shifts of 3 and 5 days (not shown) the barycenter of the histogram is slightly shifted to negative values. This is in consistence with the histogram (with very few points) of the combined 6 FD events (not shown), where from 0 to 5 days time shift, the barycenter also moves to the left. However, these shifted barycenter are not significantly different from the barycenter without time shift.

\subsection{The $\log (\mathbf{E x t})-\mathrm{CNM}$ Correlation}

[56] The extinction is influenced by both, aerosol and thin cloud load. At low altitudes, the variation of $\log (\mathrm{Ext})$ should be dominated by clouds and hence, deliver a similar (but inverse) correlation pattern in comparison to CI. The additional advantage from the $\log (\mathrm{Ext})-\mathrm{CNM}$ correlation analysis can be seen in the analysis of higher altitudes, where the extinction data are solely sensitive to enhancement in the aerosol amount.

[57] The correlation coefficients of the whole time series are shown in Figure 11. Here, blue points indicate a negative correlation and red points a positive correlation. For 0,1 and 3 days time shift, the $\log (\mathrm{Ext})-\mathrm{CNM}$ correlation possesses a marginally significant negative correlation at $0-30^{\circ} \mathrm{N}, 15 \pm$ $1.5 \mathrm{~km}$. Additionally, after a time shift of 3 days, the data show 3 marginally significant positive correlations, two of them remaining marginally significant after a time shift of 5 days. The corresponding CI-CNM correlations are not significant, but exhibit the expected sign of the correlation (a negative CI-CNM correlation coefficient corresponds to a positive $\log (\mathrm{Ext})-\mathrm{CNM}$ correlation coefficient and vice versa).

[58] By performing a SPE analysis of the combined 6 FD events (Figure 12), without time shift the $\log (\mathrm{Ext})-\mathrm{CNM}$ correlation yields two significant correlation of $\mathrm{r}=-0.29$ at $0-30^{\circ} \mathrm{S}, 12 \pm 1.5 \mathrm{~km}$ and $\mathrm{r}=+0.25$ at $0-30^{\circ} \mathrm{N}, 12 \pm 1.5 \mathrm{~km}$ and one marginally significant negative correlation of $\mathrm{r}=$ -0.41 at $30-60^{\circ} \mathrm{S}, 18 \pm 1.5 \mathrm{~km}$ (Table 2 ). The same correlations are found for a time shift of one day, but loose significance at 3 days time shift. Instead, at 3 days time shift two new marginally significant negative correlations of $\mathrm{r}=$ -0.20 at $60-90^{\circ} \mathrm{N}, 12 \pm 1.5 \mathrm{~km}$ and $\mathrm{r}=-0.25$ at $0-30^{\circ} \mathrm{N}$, $15 \pm 1.5 \mathrm{~km}$ emerge. The latter correlation is also seen in the correlation with the whole time series and in the CICNM-correlation with the expected contrary relation (see above). For a time shift of 3 days, the distribution of the correlation coefficients of the whole time series looks very similar to the distribution of the combined 6 FD events. Hence, in this case, the correlation between $\log ($ Ext $)$ and CNM of the 6 investigated FD events dominates the whole time series.

[59] Again, we partitioned the latitude bands also in longitudinal sections of $90^{\circ}$ (Figure 13). The distribution of the $\log ($ Ext)-CNM correlation coefficients resembles the (inverted) CI-CNM-correlations, hence, both possess similar cloud (or cloud/aerosol)-CNM correlations. However, the $\log (\mathrm{Ext})-$
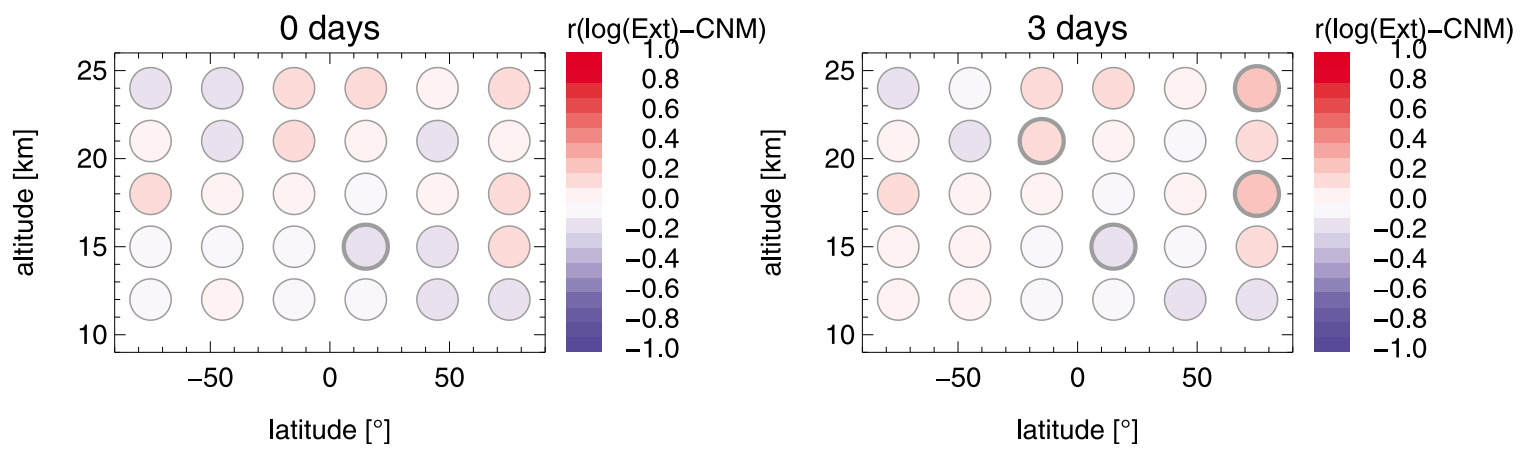

Figure 11. Latitude-altitude distribution of the correlation coefficient $r$ between $\log (\mathrm{Ext})$ and CNM for the whole time series with (left) 0 and (right) 3 days time shift. 

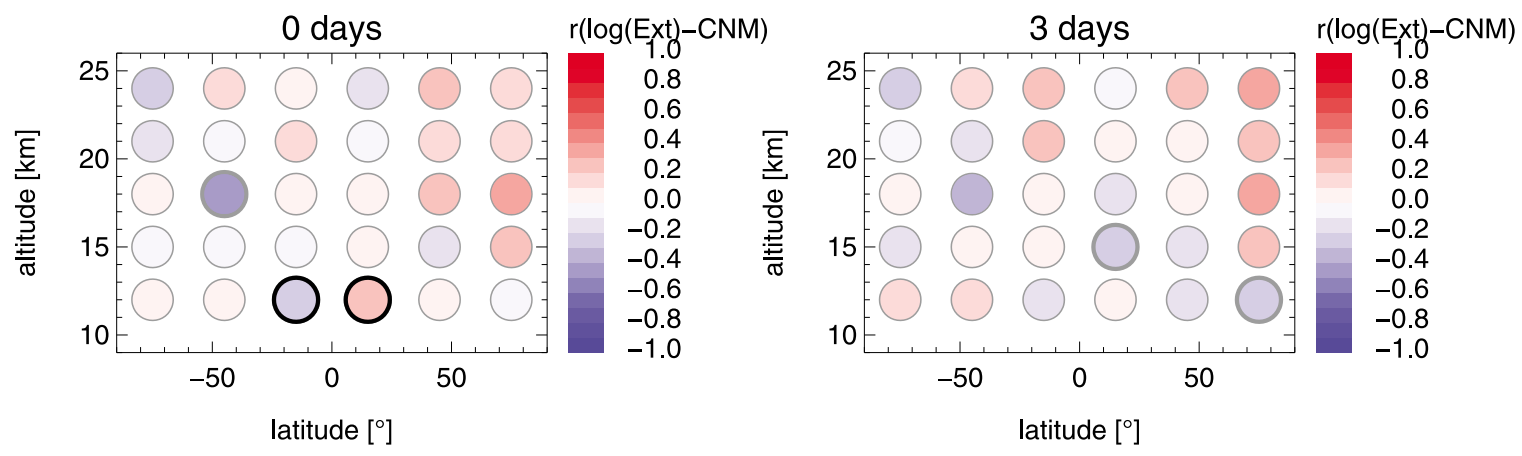

Figure 12. Same as Figure 11 but for the combined 6 FD events at 0 and 3 days time shift.

CNM correlations have slightly more negative values. In term of spatial areas the ratio of positive to negative correlations is 1.5 for the latitude-altitude bins and 0.9 for the latitudelongitude-altitude bins.

[60] During the FD event of 2003-06-22 (Figure 14, left), primarily, negative correlations are observed which diminish during the following days. Remarkably is also the FD event of 2002-07-29 (Figure 14, right) which after a time shift of 3 days possesses only positive (or neutral) correlations, 6 of them marginally significant and 2 significant. The CI at this day (not shown) also exhibits mainly the corresponding negative correlations with 2 of them being significant.

[61] The analysis of the individual events at $60-90^{\circ} \mathrm{N}$ results in 13 positive and 4 negative correlations at northern winter which changes to 4 positive and 12 negative correlations after applying a time shift of 5 days. However, more FD events are needed to analyze a possible influence of
GCRs on PSC formation. The histogram of all single events exhibits a Gaussian-like distribution (Figure 15) which is slightly (but not significantly) shifted toward positive values after 3 and 5 days time shift.

\subsection{Occ-CNM Correlation}

[62] The cloud occurrence frequency is a measure for the degree of cloudiness (percentage of cloud events with $\mathrm{CI}<$ 4.5 in a time-window of one day). In Figure 16 the results of the Occ-CNM-correlation of the whole time series is shown, with blue points for negative correlations and red points for positive correlations. The Occ-CNM-analysis without time shift exhibits a significant negative correlation at $60-90^{\circ} \mathrm{N}$, $18 \pm 1.5 \mathrm{~km}$ which persists until a time shift of 5 days. Since for this correlation, there are no data at the time periods of the investigated FD-events, the correlation for the whole time range is generated at periods with only minor change in GCR. So, the correlation coefficient might be spurious but
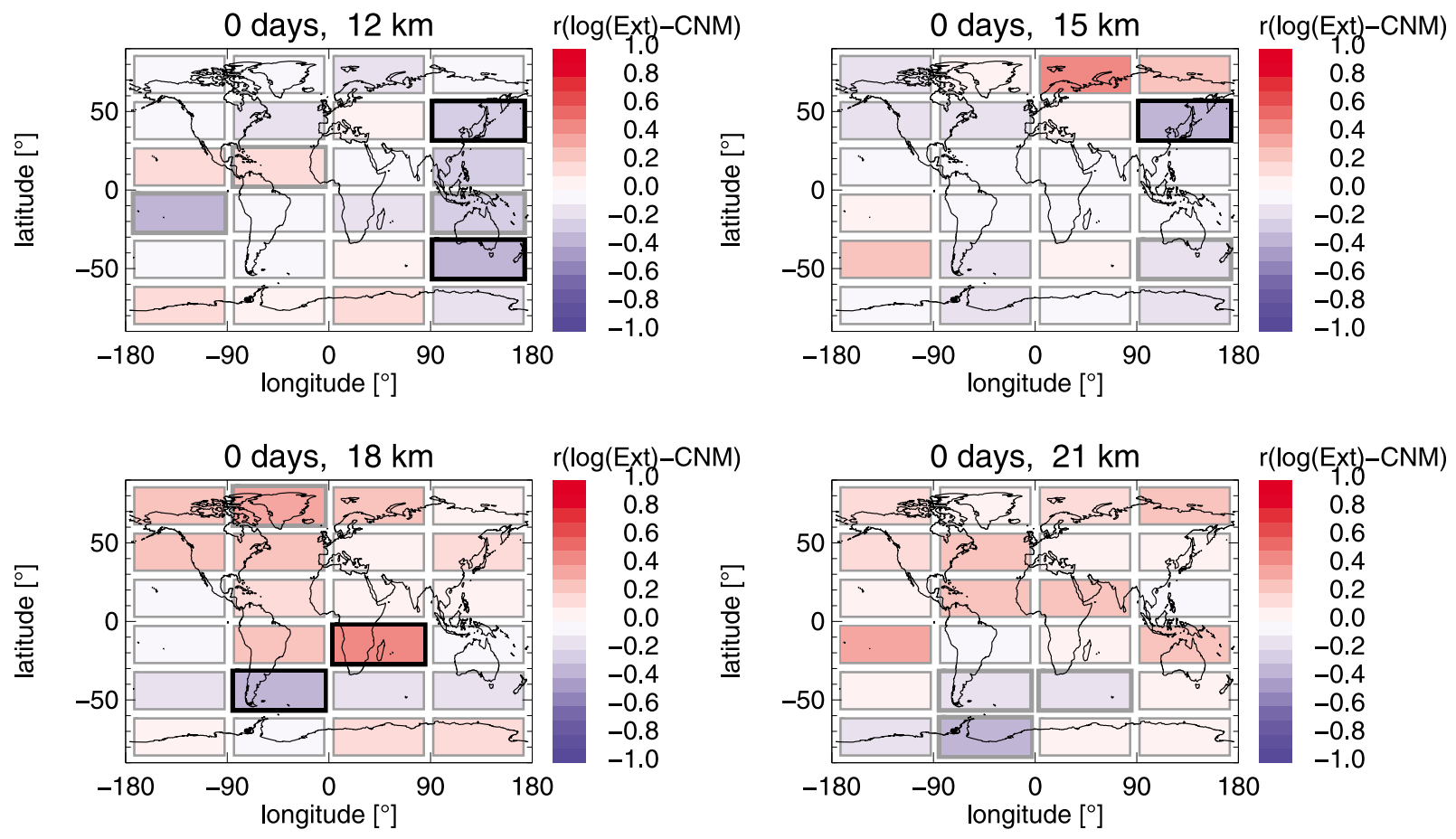

Figure 13. Latitude-longitude distribution of the correlation coefficient $r$ between $\log (E x t)$ and $C N M$ (for the $6 \mathrm{FD}$ events) at different altitudes (without time shift). 

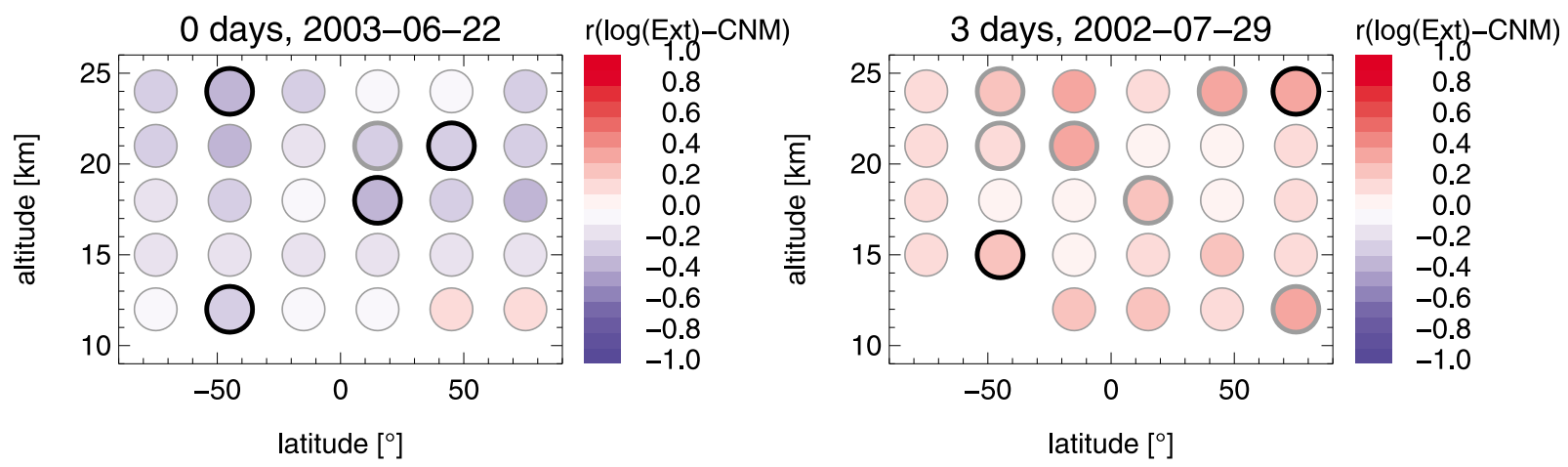

Figure 14. Same as Figure 11 but for individual FD events: (left) 2003-06-22 and (right) 2002-07-29.

also could result from minor FD events which are not included in the SPE analysis. With a time shift of 3 days we find a marginally significant positive correlation at $30-60^{\circ} \mathrm{S}$, $12 \pm 1.5 \mathrm{~km}$ and a marginally significant negative correlation at $0-30^{\circ} \mathrm{N}, 15 \pm 1.5 \mathrm{~km}$.

[63] Figure 17 and Table 2 present the results from the SPE analysis between Occ and CNM for the 6 FD events. Without time shift, there are a significant negative correlation of $\mathrm{r}=-0.65$ at $60-90^{\circ} \mathrm{S}, 12 \pm 1.5 \mathrm{~km}$ as well as two significant positive correlations of $\mathrm{r}=0.55$ at $30-60^{\circ} \mathrm{S}, 9 \pm$ $1.5 \mathrm{~km}$ and of $\mathrm{r}=+0.61$ at $30-60^{\circ} \mathrm{S}, 12 \pm 1.5 \mathrm{~km}$. The latter is also seen as negative correlation in the CI data. Additionally, there is a further marginally significant positive correlation. After a time shift of 1 day, the significant correlations are maintained and three new marginally significant correlations appear, among them the correlation at 0 $30^{\circ} \mathrm{S}, 9 \pm 1.5 \mathrm{~km}$ which is also present in the CI data. After 3 days this correlation occurs again and we find a marginally significant negative correlation at $0-30^{\circ} \mathrm{N}, 15 \pm 1.5 \mathrm{~km}$. The latter correlation is also seen in the $\mathrm{CI}$ and $\log (\mathrm{Ext})$ data.

[64] Overall, with the Occ data we find higher correlations. However, the results from the Kolmogorov-Smirnov test (Section 4.5) indicate that the Occ-CNM correlations are less reliable than the CI- and $\log (\mathrm{Ext})-\mathrm{CNM}$-correlations. This might be due to the fact that we have used daily means of the Occ data and, hence, have only 21 data points for each individual correlation. The other 2 data sets use 84 data points for each calculation.

[65] The main picture from the occurrence frequency data is very similar to the picture from the Cloud index data with an excess of positive correlations of 1.9 (in term of spatial area). Again, these data are not independent, because, normally, an increasing CI corresponds to a decreasing Occ.

[66] The refined analysis with the latitude-longitudealtitude grid of $30^{\circ} \times 90^{\circ} \times 3 \mathrm{~km}$ delivers a rather stochastical distribution of positive and negative correlations which is not in concordance with the results from the latitude-altitude-grid. We attribute this also to the reduced number of data points, so that the analysis is at the statistical limit and will not be interpreted here.

[67] Again, we performed the correlation analysis for the individual FD events. This did not produce results for all latitude-altitude intervals, because sometimes, all data points have a cloud occurrence of $0 \%$ or $100 \%$, so that no correlation analysis is possible. A noticeable feature is that for low altitudes positive correlations prevail (see FD event from 2004 to
01-09 in Figure 18). Altogether, without time shift, the individual events reveal a nearly equal amount of negative and positive correlations. This can also be seen in the histogram (Figure 19), where $r$ is distributed randomly around zero. A time shift of 1-5 days does not change the barycenter of the histogram significantly. The broad scattering of $r$ is attributed to the low number of data points.

\section{Discussion}

[68] In this Section, we investigate, whether the retrieved results are in accordance with the expected correlation for the UT/LS region. We look more precisely at the achieved dependencies of the correlation from altitude and geographical location. Initially, we confine on the analyses without time shift. Then, we test the impact of a time shift and make some estimates about the significance and the impact of the retrieved results.

\subsection{Cloud-CNM Correlation in the UT/LS}

[69] For the upper troposphere (UT), not only the magnitude, but even the sign of a possible GCR-cloud correlation is not clear - unlike the situation in the lower troposphere. As mentioned before, $Y u$ [2002] expects a decrease in aerosol production with increasing ionization. This theory is sup-

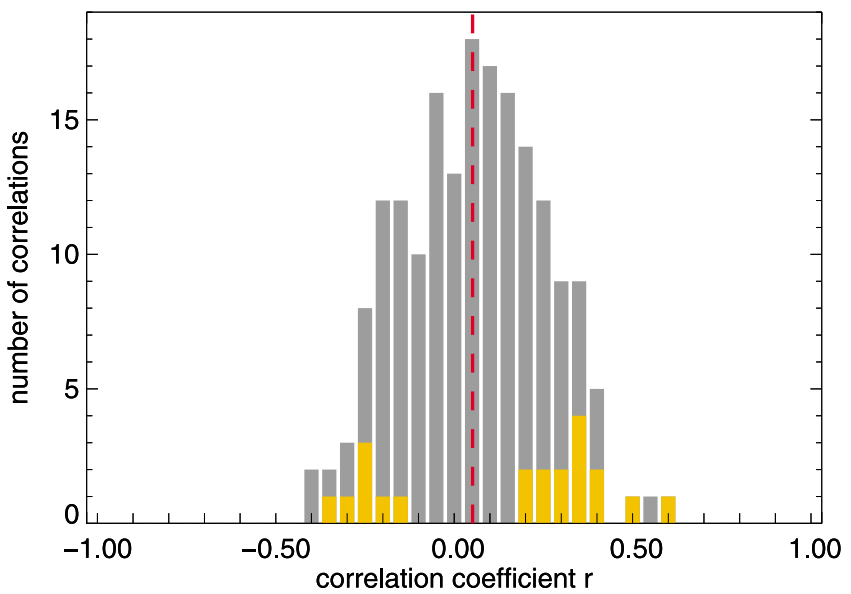

Figure 15. Histogram of correlation coefficients $r$ between $\log (\mathrm{Ext})$ and CNM for all 6 FD events (all latitudes and altitudes, 0 days time shift); orange color indicates $\mathrm{p}<0.1$; the vertical dashed line indicates the mean of $r$. 

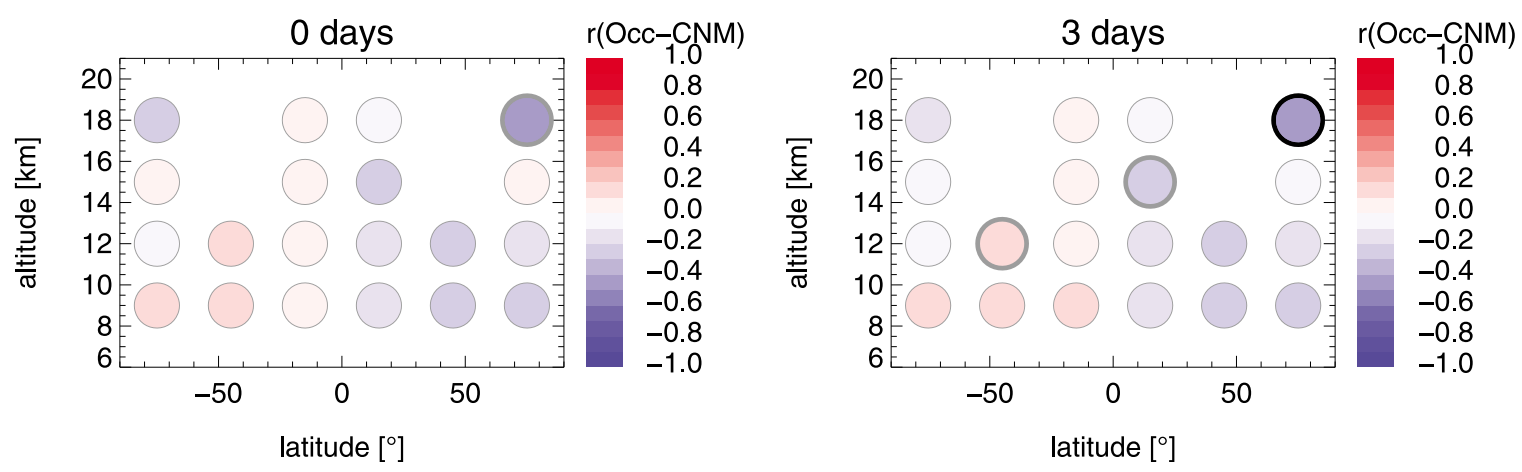

Figure 16. Latitude-altitude distribution of the correlation coefficient $r$ between Occ and CNM for the whole time series with (left) 0 and (right) 3 days time shift.

ported by the predominant negative correlation between "high clouds" from the ISCCP data set (hence, pressure levels $>440$ mbar) and cosmic rays [Voiculescu et al., 2006, 2007]. On the other hand, Eichkorn et al. [2002] found indications for ion induced aerosol nucleation in the UT and Arnold [2008] even found indications for ion induced ice nucleation. Additionally, Kazil et al. [2006] and Yu et al. [2008] have calculated that the strongest aerosol production due to ion mediated nucleation occurs in the cold tropical tropopause layer (TTL). According to this theory, a positive correlation between clouds and CNM is expected.

[70] Our findings from the CI-CNM correlation (Figure 7) and the Occ-CNM correlation (Figure 17) indicate that the sign of the effect is dependent on latitude and altitude, but that positive correlations prevail. In contrast, the $\log (\mathrm{Ext})-\mathrm{CNM}$ correlation (Figure 12) shows a more balanced picture with nearly equal amounts of positive and negative correlations.

\subsection{Altitude Dependence of the Correlation}

[71] Without time shift, in both data sets (CI and Occ) clouds and CNM are positively correlated (Figures 7 and 17) at $9 \pm 1.5 \mathrm{~km}$ altitude which is diminished or even changes the sign at $12 \pm 1.5 \mathrm{~km}$ altitude. An explanation for this weakening of the positive correlation might be that at higher altitudes, the increase in ionization rate causes a reduced lifetime of ion clusters and hence, aerosol production decreases (following the hypothesis of $Y u$ [2002]). However, at 15 and $18 \mathrm{~km}$ altitude the results are non-uniform. At high southern latitudes, there is for both, CI and Occ, a negative correlation between clouds and CNM. Contrariwise, at high northern latitudes the CI data possess a positive correlation between clouds and CNM (Occ data are not available).

[72] The altitude characteristic of the $\log ($ Ext $)$ data (Figure 12) displays a less systematic picture. At $12-18 \mathrm{~km}$ altitude it reveals a mixture of positive and negative correlations which is reasonably consistent with the findings from the CI and Occ data. At 21-24 km altitude, again the occurrence of positive and negative correlations is nearly balanced. At these high altitudes $\log (\mathrm{Ext})$ is a pure parameter for aerosols. Vanhellemont et al. [2002] describe for altitude ranges from 18.5 to $29.5 \mathrm{~km}$ significant correlations between the SAGE (Stratospheric Aerosol and Gas Experiment) and CNM data from the Climax station, but these correlations exhibit time lags of a few months. For lack of a plausible cosmic ray related mechanism and due to the autocorrelation in CNM we have not investigated such long time shifts.

\subsection{Geographical Dependence of the Correlation}

[73] In the studies which use the ISCCP data set for tropospheric clouds the sign and size of the correlations strongly depend on geographical location [Voiculescu et al., 2006]. They assume that, beside the strength of the GCR effect, the constitution and the amount of the aerosol load as well as the humidity are the reason behind this. The UT/LS, however, is less diversified in this regard. This is the reason, why we perform our main investigation for whole latitude bands. Nevertheless, also in the UT/LS there exist several different meteorological regimes. In the tropics cirrus clouds are mainly produced by large-scale upwelling and outflow of anvils. At midlatitudes and in polar regions cirrus clouds are
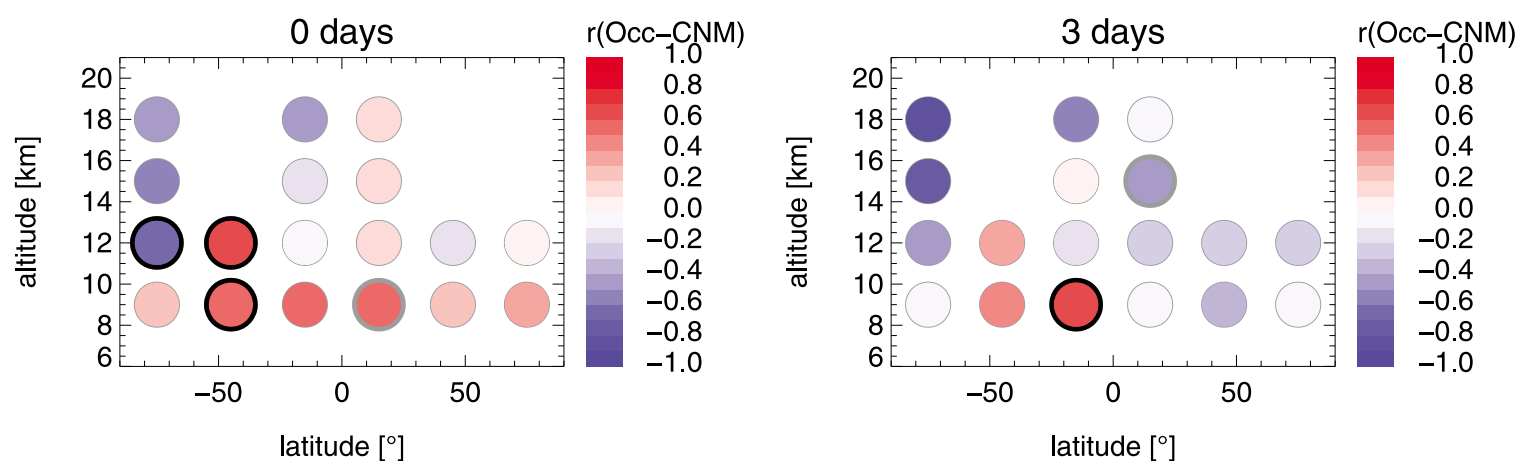

Figure 17. Same as Figure 16 but for the combined 6 FD events at 0 and 3 days time shift. 


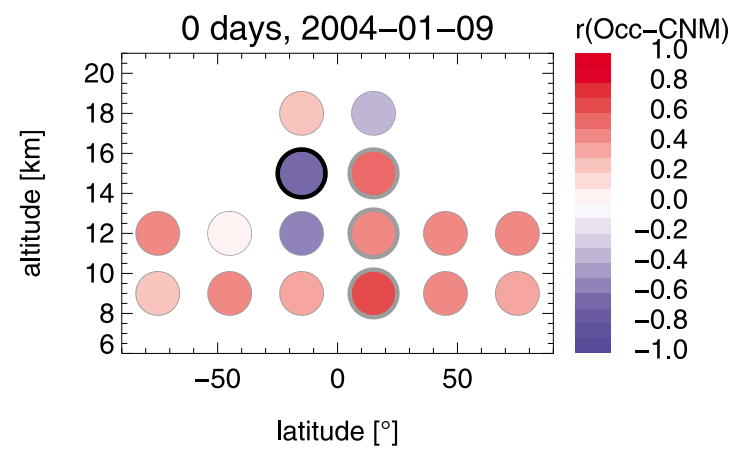

Figure 18. Same as Figure 16 but for FD at 2004-01-09.

generated by meridional transport of tropical air masses as well as in the vicinity of fronts accompanied with weather systems. In addition, in polar winter at very cold temperatures, PSCs can form.

[74] Counting the significant correlations (with $p<0.1$ ) from the correlations of the combined 6 FD events from all 3 MIPAS cloud parameters with CNM, delivers 10 significant correlations which are distributed to almost all latitudes (Figures 7, 12, and 17). Four of them occur in the tropics, among them 3 with the expected positive sign (for the cloud-CNM correlation). Actually, the cosmic ray intensity and variation at the equator is much lower than at the poles, so in this regard, an effect from GCR on high tropical clouds is unanticipated. However, by investigating the GCR-cloud link with data from the ISCCP data set, Voiculescu et al. [2006, 2007] also find significant (positive and negative) correlations between CNM and cloud cover in tropical regions.

[75] At southern midlatitudes there are also 4 significant correlations (Figures 7, 12, and 17). The CI and Occ data exhibit a positive correlation between cloud amount and CNM at $9 \pm 1.5 \mathrm{~km}$ and the Occ data also at $12 \pm 1.5 \mathrm{~km}$. At $18 \pm 1.5 \mathrm{~km}$ the $\log (\mathrm{Ext})$ data are negatively correlated with CNM. Again this can be attributed to the high altitude, where an increase in GCRs might result in a decrease in aerosol production $[Y u, 2002]$. At northern midlatitudes no significant correlation was found. An explanation might be that in the more polluted northern hemisphere the IIN effect is superimposed by the higher aerosol load of the air.

[76] At high latitudes, no clear picture emerges. Concerning polar stratospheric clouds (PSC), the data set is very sparse, as the analysis has to be confined to the short periods when PSCs are present. We would therefore need more FD events to draw any conclusion concerning the formation of PSCs due to GCRs.

[77] The latitude-longitude grids show higher correlations between clouds and CNM in some regions. An interesting feature is that in the region of the Western Pacific a negative correlation between cloud cover and CNM and between aerosols and/or clouds and CNM is found (Figures 8 and 13). This supports the findings from Voiculescu et al. [2006], who detect an anticorrelation between high cloud amount and the cosmic ray induced ionization over wide ranges of the Pacific, though our analysis is at the statistical limit.

\subsection{Time Shift Dependence of the Correlation}

[78] A cloud or aerosol occurrence might need some days to respond to a change in GCRs or solar particles. For example, Mironova et al. [2008] monitored with the TOMS satellite instrument an increase of sulfate or nitrate aerosol on the second day after a SP event in the Antarctic region.

[79] Thus, we also investigated time shifts of $0-5$ days (Section 2.3.2). An example for a new negative correlation between clouds and CNM emerging after 3-5 days is found at $0-30^{\circ} \mathrm{N}, 15 \pm 1.5 \mathrm{~km}$ ((Figures 7,12 , and 17). It is marginally significant in the $\log (\mathrm{Ext})-\mathrm{CNM}$ and Occ-CNM correlations and significant in the CI-CNM correlation. This is the only correlation which is significant $(\mathrm{p}<0.1)$ for all cloud parameter-CNM correlations. Overall, some correlations increase in the course from 0 to 3 days, whereas others disappear. The histograms of all data from the individual FD events indicate a weak enhancement of the GCR-cloud link in the MIPAS cloud parameters after a time shift from 1 to 5 days. However, this enhancement is not significant.

\subsection{Significance of the Results}

[80] In the investigated time period, 6 distinct FD events occurred. Due to the good temporal and spatial resolution of the MIPAS measurements, we compensate the low number of events by the large amount of single data points scattered over the whole latitude band. So, in spite of the low number of FD events, there are a large number of meteorological conditions included in our analysis. Overall, for the combined 6 FD events, more than 150000 data points are incorporated in the calculation of the correlation coefficients. A drawback is that the studied FD events are rather weak (6-7\% decrease in comparison to the 100 day running mean), thus the impact on cloudiness might be weak. Certainly, with more events the statistical analysis can be further improved, especially for the analysis of the polar regions as well as for the latitudelongitude distribution of the correlation coefficients.

[81] The obtained correlations are weak and most of the pvalues of the significant correlation coefficients are near the chosen significance value. According to the definition, correlating random data results in $10 \%$ correlations with $\mathrm{p}<0.1$. Therefore, it might be possible that the cloud-CNM correlations are significant by chance and not by an existing causal dependency. So, albeit we find some features which are in

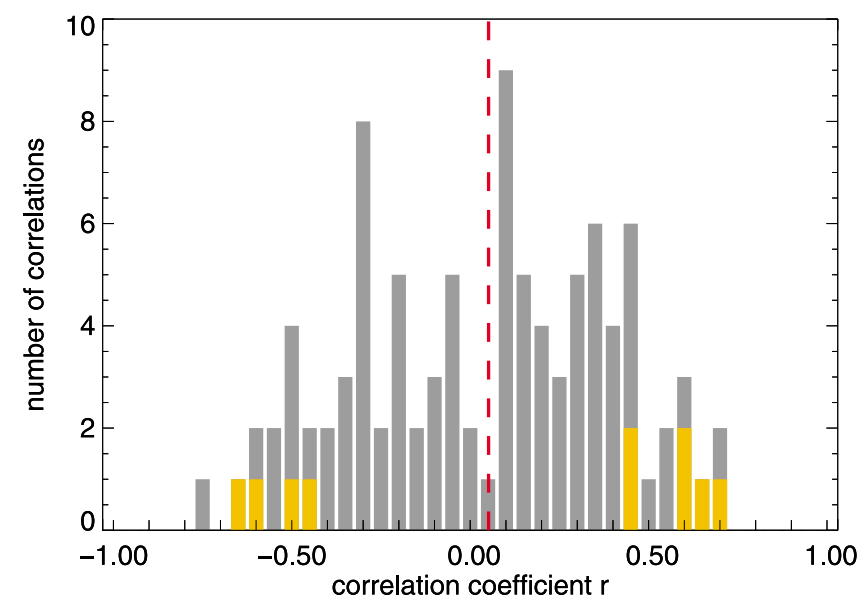

Figure 19. Histogram of correlation coefficients $r$ between Occ and CNM for all 6 FD events (all latitudes and altitudes, 0 days time shift); orange color indicates $\mathrm{p}<0.1$; the vertical dashed line indicates the mean of $r$. 

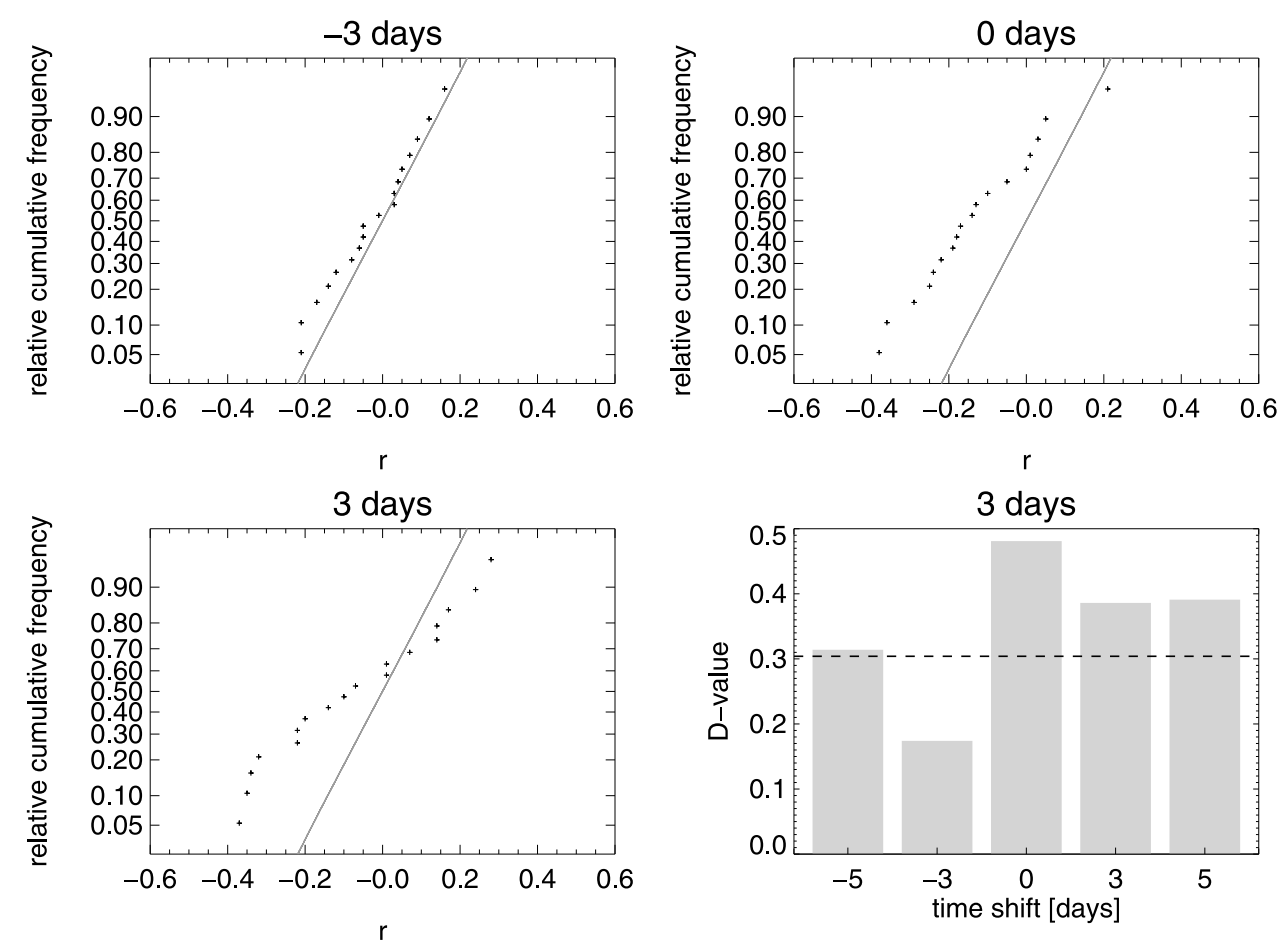

Figure 20. Relative cumulative frequency of the CI-CNM correlation coefficients obtained from the 6 combined FD events for (top left) -3 days, (top right) 0 days, and (bottom left) 3 days; the solid line indicates a random distribution. (bottom right) Kolmogorov-Smirnov test statistics D for a confidence level of $95 \%$ for the CI-CNM correlations of the 6 combined FD events for time shifts from -5 to 5 days. The critical D-value for $\mathrm{CI}$ is $\mathrm{D}_{\text {crit }}=0.304$.

accordance with the theory, like the primarily positive correlation between clouds and CNM, it is crucial to assess, whether the obtained correlations are robust enough to support the GCR-cloud hypothesis.

[82] As a first test, we implemented negative time shifts to the data. With time shifts of $-5,-3$, and -1 days, we also find some significant correlations which hypothetically would imply a reverse causality between FD events and clouds. However, the obtained quantity of significant correlations $(p<0.1)$ is severely reduced in comparison with the physical reasonable time shifts of 0,1 , and 3 days. For the CI-CNM correlation with $-5,-3$, and -1 days we find 2 correlations with $\mathrm{p}<0.1$, whereas we find 10 correlations for 0,1 , and 3 days time shift. For $\log (\mathrm{Ext})$ the ratio is $4: 8$ and for Occ 1:12. This is a first argument that the distribution of the correlation coefficients is significantly different from a random distribution. However, we should not over-interpret the obtained individual correlations, because some of them might be artifacts.

[83] In this paper we use the neutron count data (CNM) from the midlatitude cosmic ray station Climax as a proxy for the GCR induced ionization in the atmosphere. Extending the analysis to the neutron counts (HNM) from the HALEAKALA station does provide similar results (see Figure S2 in the auxiliary material). However, the ionization rate depends on latitude and altitude. Therefore, using neutron counts will always be a simplification which produces an inaccuracy and affects the significance of the various correlations and sensitivities. While computing cosmic ray induced ionization rates with detailed models is beyond the scope of this paper, we estimate the local ionization rates with a simple parameterization scheme. The latitude modulation and the solar modulation were calculated according to Hensen and van der Hage [1994] and for the altitude modulation we use the calculation according to Bazilevskaya et al. [2008]. With this rather simple parametrization the most important dependencies of the ionization rate are considered.

[84] As a second significance test we then calculate the correlation coefficients between the estimated ionization rates and the various cloud parameters as well as the corresponding sensitivities. The correlation between CI and the estimated ionization rate looks very similar to the $\mathrm{CI}-\mathrm{CNM}$-correlation (Figure S3a in the auxiliary material) with a slight tendency toward more positive values (corresponding to more negative values for the cloud-GCR correlation). The largest changes occur in the tropics which is not surprising because, there, the latitude dependency of the ionization rate has the steepest gradient. Smaller changes of $r$ are obtained for $\log (\mathrm{Ext})$ and Occ (not shown). The effect of using the ionization rate instead of the CNM data for the calculation of the impact of GCRs on clouds is shown in the next Section (4.6).

[85] As a third test we performed a Kolmogorov-Smirnov test (Section 2.3.4) on the calculated CI-CNM correlation coefficients of all latitudes and altitudes for the combined 6 FD events with time shifts from -5 to 5 days (Table 3 ). Figure 20 (top left, top right, and bottom left) shows the cumulative fraction of the obtained correlation coefficients for $-3,0$, and 3 days time shift. The solid line results from random numbers. The obtained D-values (see Table 3 and 
Table 3. Kolmogorov-Smirnov Test Statistics D for a Confidence Level of $95 \%$ for the CI-, $\log ($ Ext)-, and Occ-CNM Correlations of the Six Combined FD Events for Time Shifts From -5 to 5 Days $^{\mathrm{a}}$

\begin{tabular}{cccc}
\hline Time Shift (days) & $\mathrm{D}(\mathrm{CI})$ & $\mathrm{D}(\log ($ Ext $))$ & $\mathrm{D}(\mathrm{Occ})$ \\
\hline-5 & 0.314 & 0.308 & $>1.00$ \\
-3 & 0.174 & 0.155 & 0.293 \\
0 & 0.481 & $>1.00$ & $>1.00$ \\
3 & 0.386 & $>1.00$ & $>1.00$ \\
5 & 0.391 & $>1.00$ & 0.398 \\
\hline
\end{tabular}

${ }^{a}$ The critical D-value for CI and Occ is $D_{\text {crit }}=0.304$ and for $\log ($ Ext $)$ $\mathrm{D}_{\text {crit }}=0.248$.

Figure 20 (bottom right)) represent the highest vertical distance between the two curves.

[86] For the CI and Occ data with $N=20$, we utilized the $5 \%$ critical D-value of $\mathrm{D}_{\text {crit. }}=0.304$ and for the $\log (\mathrm{Ext})$ data with $\mathrm{N}=30$ a value of $\mathrm{D}_{\text {crit. }}=0.248$. If the $\mathrm{D}$-value is above $\mathrm{D}_{\text {crit }}$, the null hypothesis (the distribution of observed correlation coefficients is equal to the distribution of correlation coefficients from random numbers) is rejected. With exception of a time shift of -5 days, where D is slightly above $\mathrm{D}_{\text {crit }}$, all correlation analysis with negative time shifts reveal $\mathrm{D}$-values lower than $\mathrm{D}_{\text {crit }}$. For 0 to +5 days time shift, however, D-values above $\mathrm{D}_{\text {crit }}$ are obtained. The same result is obtained with the $\log (\mathrm{Ext})$ data.

[87] Thus, for the CI-CNM correlation, we derive a probability of more than $95 \%$ that for positive time shifts between 0 and 5 days the entity of the correlation coefficients is different from the null hypothesis. Therefore, there is strong statistical evidence that for these cases, the obtained distribution of $\mathrm{r}$ differs significantly from the null hypothesis and is not coincidental. Negative time shifts do not result in a significant correlation. For the Occ-CNM correlation, the Kolmogorov-Smirnov test also results in a rejection of the null hypothesis for $0-5$ days time shift, but also for -5 and -1 days. So, the results from Occ are less explicit.

\subsection{Estimation of the Effect of GCRs on CI and $\log (\mathrm{Ext})$}

[88] We can estimate the confidence intervals of a possible impact from GCRs on the Cloud Index (CI) and the logarithm of the extinction $\log (\mathrm{Ext})$ for the investigated altitude intervals. For this purpose we calculate the correlation coefficients for the $6 \mathrm{FD}$ events between $\mathrm{CI}$ and $\mathrm{CNM}$ at 9 and $12 \mathrm{~km}$ altitude and between $\log (\mathrm{Ext})$ and CNM at all altitude intervals but without partitioning of the latitude or longitude. For the correlation of CI and CNM at 15 and $18 \mathrm{~km}$ altitude, we restrict the latitude range to $-30-30^{\circ} \mathrm{N}$, where high clouds exist. The obtained correlation coefficients for the individual altitudes are not significant. Nevertheless, it is possible to use them for the calculation of a confidence interval of the impact of GCRs on CI and $\log (\mathrm{Ext})$.

[89] From the obtained correlation coefficient $r$ and the mean values of the cloud parameter and CNM, as well as their standard deviations $\sigma$ we estimate the sensitivity $\mathrm{S}$ of the CI-CNM and $\log (\mathrm{Ext})-\mathrm{CNM}$ relation.

$$
S \pm \sigma(S)=(r \pm \sigma(r)) *\left(\frac{\sigma(\text { cloud parameter })}{\overline{\text { cloud parameter }}}\right) /\left(\frac{\sigma(C N M)}{\overline{C N M}}\right)
$$

[90] The obtained results are presented in Table 4 and in Figure 21. The highest sensitivities are found at low altitudes. At $9 \mathrm{~km}$ altitude the correlation coefficient of the CICNM relation is $\mathrm{r}=-0.11 \pm 0.12(1 \sigma)$. The calculation of the best estimate of the sensitivity yields $\mathrm{S}_{\text {best }}=-28 \%$ with $\mathrm{S}_{\min }(1 \sigma)=-58 \%$ and $\mathrm{S}_{\max }=+3 \%$. That means that, for example, an increase in GCRs which produces a $15 \%$ increase in CNM (typical amplitude of CNM for a solar cycle) would at $9 \mathrm{~km}$ altitude lead to a change in CI between $-9 \%$ and $+0.5 \%$ with a best estimate of $-4.1 \%$. At $12 \mathrm{~km}$ altitude the sensitivity is $-13 \%(-40-13 \%)$. At higher altitudes the calculation of the sensitivity is restricted to $-30-30^{\circ} \mathrm{N}$ latitude. Here, we obtain at $15 \mathrm{~km}$ altitude only a weak sensitivity $\left(\mathrm{S}_{\text {best }}=-10 \%(-39-20 \%)\right)$. At $18 \mathrm{~km}$, however, the sensitivity is $\mathrm{S}_{\text {best }}=-65 \%$ with $\mathrm{S}_{\min }=-168 \%$ and $\mathrm{S}_{\max }=37 \%$. Despite the broad range of the sensitivity, this finding provides an indication for a positive GCR-cloud feedback in the TTL.

[91] For $\log ($ Ext $)$ the best estimate of the sensitivity at $12 \mathrm{~km}$ altitude is $\mathrm{S}_{\text {best }}=-16 \%$ with $\mathrm{S}_{\min }=-32 \%$ and $\mathrm{S}_{\max }=$ $0 \%$. So, an $15 \%$ increase in CNM would lead to a $-2.4 \%$ $(-5-0 \%)$ decrease in $\log (\mathrm{Ext})$ at $12 \mathrm{~km}$ altitude. This is a discrepancy to the findings from the CI-CNM correlation. At higher altitudes the sensitivity of the $\log (\mathrm{Ext})-\mathrm{CNM}$ effect shifts toward weak positive values which is in accordance to the findings from the CI-CNM analysis.

[92] Using the HNM data delivers larger sensitivities but also larger error bars (not shown). So, the HALEAKALA data displays the same picture of the GCR-cloud effect, but the Climax data is more appropriate for the analysis.

[93] Finally, using the inferred ionization rate instead of the CNM data for the calculation of the sensitivity produces very similar results (Figure S3b in the auxiliary material) for the whole latitude ranges. The reason might be that both, the cosmic ray induced ionization and the neutron monitor variations during FD events, are defined by essentially the same process - modulation of the cosmic ray spectrum near Earth by the interplanetary transient. A difference is mainly seen in the tropics, where at $12 \mathrm{~km}$ altitude the sensitivity (concerning the cloud opacity-GCR link) is positive for CI$\mathrm{CNM}$ but negative for CI-ionization rate. This is caused by

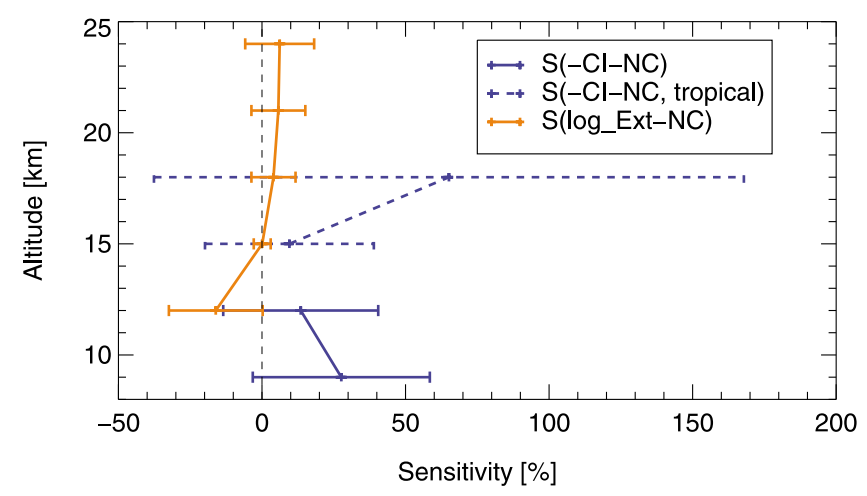

Figure 21. Sensitivity $\mathrm{S}$ of the impact of GCRs on the cloud parameters $\mathrm{CI}$ and $\log (\mathrm{Ext})$; latitudinal range for $\mathrm{CI}$ at $9,12 \mathrm{~km}$ altitude: $-90-90^{\circ} \mathrm{N}$, at $15,18 \mathrm{~km}$ altitude: $-30-30^{\circ} \mathrm{N}$; latitudinal range for $\log (\mathrm{Ext})$ at all altitudes: $-90-90^{\circ} \mathrm{N}$. 
Table 4. Estimation of the Sensitivity $\mathrm{S}$ of the Impact of GCRs on the Cloud Parameters CI and $\log (\operatorname{Ext})^{\mathrm{a}}$

\begin{tabular}{|c|c|c|c|c|c|c|}
\hline Altitude (km) & Mean (var) & Mean CNM (counts per hour) & $\mathrm{r}$ & $\mathrm{S}_{\text {best }}$ & $\mathrm{S}_{\min }(1 \sigma)$ & $\mathrm{S}_{\max }(1 \sigma)$ \\
\hline \multicolumn{7}{|c|}{$V a r=C I$} \\
\hline 9 & 3.43 & 3606 & -0.112 & -0.276 & -0.585 & 0.032 \\
\hline 12 & 5.03 & 3606 & -0.071 & -0.135 & -0.405 & 0.135 \\
\hline 15 & 4.97 & 3606 & -0.038 & -0.096 & -0.390 & 0.199 \\
\hline 18 & 6.17 & 3606 & -0.199 & -0.651 & -1.679 & 0.373 \\
\hline \multicolumn{7}{|c|}{ Var $=\log (E x t)$} \\
\hline 12 & -4.34 & 3609 & -0.206 & -0.161 & -0.324 & 0.002 \\
\hline 15 & -4.42 & 3609 & 0.002 & 0.000 & -0.029 & 0.030 \\
\hline 18 & -4.50 & 3609 & 0.099 & 0.040 & -0.037 & 0.117 \\
\hline 21 & 4.76 & 3609 & 0.127 & 0.057 & -0.037 & 0.151 \\
\hline 24 & -4.99 & 3609 & 0.172 & 0.062 & -0.058 & 0.182 \\
\hline
\end{tabular}

${ }^{a}$ Latitudinal range for $\mathrm{CI}$ at $9,12 \mathrm{~km}$ altitude: $-90-90^{\circ} \mathrm{N}$, at $15,18 \mathrm{~km}$ altitude: $-30-30^{\circ} \mathrm{N}$; latitudinal range for $\log \left(\right.$ Ext) at all altitudes: $-90-90^{\circ} \mathrm{N}$.

the steep gradient of the ionization rate with latitude in the tropics. Here, more FD events are needed to estimate the sensitivity. Overall, the usage of the inferred ionization rate instead of the directly observed CNM data does not improve the result of the analysis, maybe because of the inadequateness of the simple parametrization or due to opposing aerosol formation processes triggered by it.

\section{Summary}

[94] Until now, the GCR-cloud link is mainly found in the ISCCP-data set which tends to overlook subvisible cirrus. In the present study, we used different cloud parameters (cloud index, cloud occurrence frequency and extinction data) from the limb viewing satellite instrument MIPAS-E from July 2002 until April 2004. Due to the good vertical resolution and the high sensitivity for optically thin clouds, MIPAS is especially suited for the investigation of the GCR-cloud link at high altitudes. We focus on the investigation of shortterm changes in GCRs associated with FD events. This has the advantage that a possible GCR-cloud link cannot be superimposed by other climate variability like ENSO. During the investigated period, 6 major Forbush decrease (FD) events occurred. Due to the large amount and global coverage of MIPAS-E measurements, we can compensate the low number of events.

[95] We applied several independent methods like correlating the MIPAS-E cloud parameter from the whole time series, in a SPE analysis with 6 FD events, and from the individual FD events with the CNM data from the Climax station.

[96] Our findings indicate that a positive correlation between clouds and CNM prevails and that this correlation is especially pronounced at $9 \mathrm{~km}$. In the $\log (\mathrm{Ext})$ data at 21 and $24 \mathrm{~km}$ altitude, positive and negative correlations occur in nearly equal amounts. No clear dependence of the GCRcloud link on latitude is detectable. Applying a time shift of 0 to +5 days to the data results in slightly higher correlations, but this enhancement is not significant. With the Kolmogorov-Smirnov test we prove that for time shifts from 0 to +5 days the distribution of the obtained correlation coefficients is significantly different from the null hypothesis, whereas this is not the case for time shifts from -3 to -1 days. Hence, we draw the conclusion that the weak GCR - high cloud (or high cloud plus aerosol) effect in the MIPAS-E measurements is real and not coincidental.
[97] During one solar cycle, a typical amplitude of the Climax neutron counts is $15 \%$. By calculating the impact from a $15 \%$ increase in CNM on clouds from the MIPAS-E measurements we derive small decreases in CI (which has low values for optically thick clouds). This effect is not significant at the $1 \sigma$-level. However, the most pronounced sensitivity exists at $9 \mathrm{~km}$ altitude $(-9 \%$ to $+0.5 \%)$. For the $\log ($ Ext) data at $12 \mathrm{~km}$ altitude a decrease of -5 to $0 \%$ is calculated. At higher altitudes the effect in $\log ($ Ext) shifts toward weak positive values. The quality of the Occ-CNM correlations is not sufficient to estimate the GCR-Occ impact. Further investigations with a prolonged MIPAS data set are needed to narrow down the range of a possible GCR mid and high cloud effect. However, albeit a zero-effect cannot be excluded and the range of the estimated values is quite broad, the best estimate of the CI-CNM analyses indicates that an increase in GCRs leads to a small increase in mid and high cloud cover.

[98] Acknowledgments. We thank the National Science Foundation grant ATM-0339527 for providing the Climax-Neutron count data and ESA for the preparation of the MIPAS level $1 \mathrm{~b}$ and level 2 data. We also thank our colleague Lars Hoffmann for the development of the aerosol extinction retrieval and excellent support for handling the JURASSIC program package. This work is funded by the Deutsche Forschungsgemeinschaft under the CAWSES SPP grant (SAGACITY).

\section{References}

Arnold, F. (2008), Atmospheric ions and aerosol formation, Space Sci. Rev., 137, 225-239, doi:10.1007/s11214-008-9390-8.

Bazilevskaya, G. A., et al. (2008), Cosmic ray induced ion production in the atmosphere, Space Sci. Rev., 137, 149-173, doi:10.1007/s11214008-9339-y.

Brandt, S. (1999), Data Analysis-Statistical and Computational Methods for Scientists and Engineers, 686 pp., Springer, Berlin.

Curtius, J., E. R. Lovejoy, and K. D. Froyd (2006), Atmospheric ioninduced aerosol nucleation, Space Sci. Rev., 125, 159-167, doi:10.1007/s11214-006-9054-5.

Ebisuzaki, W. (1997), A method to estimate the statistical significance of a correlation when the data are serially correlated, J. Clim., 10, 2147-2153, doi:10.1175/1520-0442(1997)010<2147:AMTETS>2.0.CO;2.

Eichkorn, S., S. Wilhelm, H. Aufmhoff, K. H. Wohlfrom, and F. Arnold (2002), Cosmic ray-induced aerosol formation: First observational evidence from aircraft-based ion mass spectrometer measurements in the upper troposphere, Geophys. Res. Lett., 29(14), 1698, doi:10.1029/ 2002GL015044

Enghoff, M. B., and H. Svensmark (2008), The role of atmospheric ions in aerosol nucleation-A review, Atmos. Chem. Phys., 8, 4911-4923, doi:10.5194/acp-8-4911-2008.

Farrar, P. D. (2000), Are cosmic rays influencing oceanic cloud coverageor is it only El Niño?, Clim. Change, 47, 7-15, doi:10.1023/ A: 1005672825112 . 
Fischer, H., and H. Oelhaf (1996), Remote sensing of vertical profiles of atmospheric trace constituents with MIPAS limb emission spectrometers, Appl. Opt., 35(16), 2787-2796, doi:10.1364/AO.35.002787.

Fischer, H., et al. (2008), MIPAS: An instrument for atmospheric and climate research, Atmos. Chem. Phys., 8, 2151-2188, doi:10.5194/acp-82151-2008.

Flückiger, E., and R. Bütikofer (2004), Neutron monitors-Study of solar and galactic cosmic rays, Activity Rep. 2003, Int. Found. HFSJG, Bern.

Froyd, K. D., and E. R. Lovejoy (2003a), Experimental thermodynamics of cluster ions composed of $\mathrm{H}_{2} \mathrm{SO}_{4}$ and $\mathrm{H}_{2} \mathrm{O}$. 1. Positive ions, J. Phys. Chem., 107, 9800-9811.

Froyd, K. D., and E. R. Lovejoy (2003b), Experimental thermodynamics of cluster ions composed of $\mathrm{H}_{2} \mathrm{SO}_{4}$ and $\mathrm{H}_{2} \mathrm{O}$. 2. Measurements and ab initio structures of negative ions, J. Phys. Chem., 107, 9812-9824.

Greenhough, J., J. J. Remedios, H. Sembhi, and L. J. Kramer (2005), Towards cloud detection and cloud frequency distributions from MIPAS infra-red observations, Adv. Space Res., 36, 800-806, doi:10.1016/j. asr.2005.04.096.

Harrison, R. G., and D. B. Stephenson (2006), Empirical evidence for a nonlinear effect of galactic cosmic rays on clouds, Proc. R. Soc. A., 462, 1221-1233, doi:10.1098/rspa.2005.1628.

Hensen, A., and J. C. H. van der Hage (1994), Parameterization of cosmic radiation at sea level, J. Geophys. Res., 99(D5), 10,693-10,695, doi:10.1029/93JD01226.

Hervig, M., and M. McHugh (1999), Cirrus detection using HALOE measurements, Geophys. Res. Lett., 26(6), 719-722, doi:10.1029/ 1999GL900069.

Hoffmann, L. (2006), Schnelle Spurengasretrieval für das Satellitenexperiment Envisat MIPAS, Tech. Rep. JUEL-4207, Forschungszentrum Jülich, Jülich, Germany.

Hoffmann, L., M. Kaufmann, R. Spang, R. Müller, J. J. Remedios, D. P. Moore, C. M. Volk, T. von Clarmann, and M. Riese (2008), Envisat MIPAS measurements of CFC-11: Retrieval, validation, and climatology, Atmos. Chem. Phys., 8, 3671-3688, doi:10.5194/acp-8-3671-2008.

Höpfner, M., et al. (2006a), MIPAS detects Antarctic stratospheric belt of NAT PSCs caused by mountain waves, Atmos. Chem. Phys., 6, 12211230, doi:10.5194/acp-6-1221-2006.

Höpfner, M., et al. (2006b), Spectroscopic evidence for NAT, STS, and ice in MIPAS infrared limb emission measurements of polar stratospheric clouds, Atmos. Chem. Phys., 6, 1201-1219, doi:10.5194/acp-6-12012006.

Intergovernmental Panel on Climate Change (IPCC) (2007), Climate Change 2007: The Physical Science Basis. Contribution of Working Group I to the Fourth Assessment Report of the Intergovernmental Panel on Climate Change, edited by S. Solomon et al., Cambridge Univ. Press, Cambridge, U. K.

Jorgensen, T. S., and A. W. Hansen (2000), Comments on "Variation of cosmic ray flux and global cloud coverage-A missing link in solarclimate relationships" by Henrik Svensmark and Eigil Friis-Christensen, J. Atmos. Sol. Terr. Phys., 62, 73-77, doi:10.1016/S1364-6826(99) 00106-6.

Kazil, J., and E. R. Lovejoy (2004), Tropospheric ionization and aerosol production: A model study, J. Geophys. Res., 109, D19206, doi:10.1029/ 2004JD004852.

Kazil, J., E. R. Lovejoy, M. C. Barth, and K. O’Brien (2006), Aerosol nucleation over oceans and the role of galactic cosmic rays, Atmos. Chem. Phys., 6, 4905-4924, doi:10.5194/acp-6-4905-2006.

Kazil, J., G. Harrison, and E. R. Lovejoy (2008), Tropospheric new particle formation and the role of ions, Space Sci. Rev., 137, 241-255, doi:10.1007/s11214-008-9388-2.

Kniveton, D. R. (2004), Precipitation, cloud cover and Forbush decreases in galactic cosmic rays, J. Atmos. Sol. Terr. Phys., 66, 1135-1142, doi:10.1016/j.jastp.2004.05.010.

Kristjánsson, J. E., J. Kristiansen, and E. Kaas (2004), Solar activity, cosmic rays, clouds and climate-an update, Adv. Space Res., 34, 407-415, doi:10.1016/j.asr.2003.02.040.

Kristjánsson, J. E., C. W. Stjern, F. Stordal, A. M. Fjæraa, G. Myhre, and $\mathrm{K}$. Jónasson (2008), Cosmic rays, $\mathrm{CCN}$ and clouds-a reassessment using MODIS data, Atmos. Chem. Phys. Discuss., 8, 13,265-13,299, doi:10.5194/acpd-8-13265-2008.

Lee, S.-H., J. M. Reeves, J. C. Wilson, D. E. Hunton, A. A. Viggiano, T. M. Miller, J. O. Ballenthin, and L. R. Lait (2003), Particle formation by ion nucleation in the upper troposphere and lower stratosphere, Science, 301, 1886-1889, doi:10.1126/science.1087236.

Lovejoy, E. R., J. Curtius, and K. D. Froyd (2004), Atmospheric ioninduced nucleation of sulfuric acid and water, J. Geophys. Res., 109 D08204, doi:10.1029/2003JD004460.
Marsh, N. D., and H. Svensmark (2000), Low cloud properties influenced by cosmic rays, Phys. Rev. Lett., 85, 5004-5007, doi:10.1103/PhysRevLett.85.5004.

Marsh, N. D., and H. Svensmark (2003), Galactic cosmic rays and El NiñoSouthern Oscillation trends in International Satellite Cloud Climatology Project D2 low-cloud properties, J. Geophys. Res., 108(D6), 4195, doi:10.1029/2001JD001264.

Massie, S. T., A. Gettelman, W. Randel, and D. Baumgardner (2002), Distribution of tropical cirrus in relation to convection, J. Geophys. Res., 107(D21), 4591, doi:10.1029/2001JD001293.

Mergenthaler, J. L., A. E. Roche, J. B. Kumer, and G. A. Ely (1999), Cryogenic Limb Array Etalon Spectrometer observations of tropical cirrus, $J$. Geophys. Res., 104(D18), 22,183-22,194, doi:10.1029/1999JD900397.

Mironova, I. A., L. Desorgher, I. G. Usoskin, E. O. Flückiger, and R. Bütikofer (2008), Variations of aerosol optical properties during the extreme solar event in January 2005, Geophys. Res. Lett., 35, L18610, doi:10.1029/2008GL035120.

Pallé, E. (2005), Possible satellite perspective effects on the reported correlations between solar activity and clouds, Geophys. Res. Lett., 32, L03802, doi:10.1029/2004GL021167.

Pallé, E., C. J. Butler, and K. O’Brien (2004), The possible connection between ionization in the atmosphere by cosmic rays and low level clouds, J. Atmos. Sol. Terr. Phys., 66, 1779-1790, doi:10.1016/j. jastp.2004.07.041.

Pierce, J. R., and P. J. Adams (2009), Can cosmic rays affect cloud condensation nuclei by altering new particle formation rates? Geophys. Res. Lett., 36, L09820, doi:10.1029/2009GL037946.

Pudovkin, M. I., and S. V. Veretenenko (1995), Cloudiness decreases associated with Forbush-decreases of galactic cosmic rays, J. Atmos. Terr. Phys., 57, 1349-1355, doi:10.1016/0021-9169(94)00109-2.

Rodgers, C. D. (2000), Inverse Methods of Atmospheric Sounding: Theory and Practice, Ser. on Atmos. Ocean and Planet. Phys., vol. 2, World Sci., Hackensack, N. J.

Rohrer, F., and H. Berresheim (2006), Strong correlation between levels of tropospheric hydroxyl radicals and solar ultraviolet radiation, Nature, 442, 184-187, doi:10.1038/nature04924.

Sachs, L., and J. Hedderich (2006), Angewandte Statistik-Methodensammlung mit $R, 702$ pp., Springer, Berlin.

Sloan, T., and A. W. Wolfendale (2008), Testing the proposed causal link between cosmic rays and cloud cover, Environ. Res. Lett., 3, 024001, doi:10.1088/1748-9326/3/2/024001.

Spang, R., G. Eidmann, M. Riese, D. Offermann, L. Pfister, and P. H. Wang (2002), CRISTA observations of cirrus clouds around the tropopause, J. Geophys. Res., 107(D23), 8174, doi:10.1029/2001JD000698.

Spang, R., J. J. Remedios, and M. P. Barkley (2004), Colour indices for the detection and differentiation of cloud types in infra-red limb emission spectra, Adv. Space Res., 33, 1041-1047, doi:10.1016/S0273-1177(03) 00585-4.

Spang, R., J. J. Remedios, L. J. Kramer, L. R. Poole, M. D. Fromm, M. Müller, G. Baumgarten, and P. Konopka (2005a), Polar stratospheric cloud observations by MIPAS on Envisat: Detection method, validation and analysis of the Northern Hemisphere winter 2002 2003, Atmos. Chem. Phys., 5, 679-692, doi:10.5194/acp-5-679-2005. Spang, R., J. J. Remedios, S. Tilmes, and M. Riese (2005b), MIPAS observations of polar stratospheric clouds in the Arctic 2002/3 and Antarctic 2003 winters, Adv. Space Res., 36, 868-878, doi:10.1016/j. asr.2005.03.092.

Spang, R., L. Hoffmann, S. Rohs, M. Riese, J. J. Remedios, and D. Moore (2007), MIPAS Aerosol Retrievals: Towards a global climatology of multi-wavelength extinctions and aerosol size distribution, paper presented at Envisat Symposium, Eur. Space Agency, Montreux, Switzerland.

Sun, B., and R. Bradley (2002), Solar influences on cosmic rays and cloud formation: A reassessment, J. Geophys. Res., 107(D14), 4211, doi:10.1029/2001JD000560.

Svensmark, H., and E. Friis-Christensen (1997), Variations of cosmic ray flux and global cloud coverage - a missing link in solar-climate relationships, J. Atmos. Sol. Terr. Phys., 59, 1225-1232, doi:10.1016/S13646826(97)00001-1.

Svensmark, H., J. O. P. Pedersen, N. D. Marsh, M. B. Enghoff, and U. I. Uggerhøj (2007), Experimental evidence for the role of ions in particle nucleation under atmospheric conditions, Proc. R. Soc. A, 463, 385-396, doi:10.1098/rspa.2006.1773.

Svensmark, H., T. Bondo, and J. Svensmark (2009), Cosmic ray decreases affect atmospheric aerosols and clouds, Geophys. Res. Lett., 36, L15101, doi:10.1029/2009GL038429.

Tinsley, B. A. (2000), Influence of solar wind on the global electric circuit, and inferred effects on cloud microphysics, temperature, and dynamics in 
the troposphere, Space Sci. Rev., 94, 231-258, doi:10.1023/ A: 1026775408875 .

Tinsley, B. A., and F. Yu (2004), Atmospheric ionization and clouds as links between solar activity and climate, in Solar Variability and Its Effects on Climate, Geophys. Monogr. Ser., vol. 141, edited by J. M. Pap et al., pp. 321-339, AGU, Washington, D. C.

Tinsley, B. A., G. B. Burns, and L. Zhou (2007), The role of the global electric circuit in solar and internal forcing of clouds and climate, $A d v$. Space Res., 40, 1126-1139, doi:10.1016/j.asr.2007.01.071.

Todd, M. C., and D. R. Kniveton (2001), Changes in cloud cover associated with Forbush decreases of galactic cosmic rays, J. Geophys. Res., 106(D23), 32,031-32,041, doi:10.1029/2001JD000405.

Todd, M. C., and D. R. Kniveton (2004), Short-term variability in satellitederived cloud cover and galactic cosmic rays: An update, J. Atmos. Sol. Terr. Phys., 66, 1205-1211.

Udelhofen, P. M., and R. D. Cess (2001), Cloud cover variations over the United States: An influence of cosmic rays or solar variability? Geophys. Res. Lett., 28(13), 2617-2620, doi:10.1029/2000GL012659.

Usoskin, I. G., and G. A. Kovaltsov (2008), Cosmic rays and climate of Earth: Possible connection, C. R. Geosci., 340, 441-450, doi:10.1016/j. crte.2007.11.001.

Usoskin, I. G., N. Marsh, G. A. Kovaltsov, K. Mursula, and O. G. Gladysheva (2004), Latitudinal dependence of low cloud amount on cosmic ray induced ionization, Geophys. Res. Lett., 31(16), L16109, doi:10.1029/ 2004 GL019507.

Usoskin, I. G., M. Voiculescu, G. A. Kovaltsov, and K. Mursula (2006), Correlation between clouds at different altitudes and solar activity: Fact or artifact?, J. Atmos. Sol. Terr. Phys., 68, 2164-2172, doi:10.1016/j. jastp.2006.08.005.
Vanhellemont, F., D. Fussen, and C. Bingen (2002), Cosmic rays and stratospheric aerosols: Evidence for a connection?, Geophys. Res. Lett., 29(15), 1715, doi:10.1029/2002GL015567.

Voiculescu, M., I. G. Usoskin, and K. Mursula (2006), Different response of clouds to solar input, Geophys. Res. Lett., 33, L21802, doi:10.1029/ 2006GL027820

Voiculescu, M., I. G. Usoskin, and K. Mursula (2007), Effect of ENSO and volcanic events on the Sun-cloud link, Adv. Space Res., 40, 1140-1145, doi:10.1016/j.asr.2007.01.089.

$\mathrm{Yu}, \mathrm{F}$. (2002), Altitude variations of cosmic ray induced production of aerosols: Implications for global cloudiness and climate, J. Geophys. Res., 107(A7), 1118, doi:10.1029/2001JA000248.

$\mathrm{Yu}, \mathrm{F}$., and R. P. Turco (2000), Ultrafine aerosol formation via ion-mediated nucleation, Geophys. Res. Lett., 27(6), 883-886, doi:10.1029/ 1999GL011151.

Yu, F., and R. P. Turco (2001), From molecular clusters to nanoparticles: The role of ambient ionization in tropospheric aerosol formation, $J$. Geophys. Res., 106(D5), 4797-4814, doi:10.1029/2000JD900539.

Yu, F., Z. Wang, G. Luo, and R. Turco (2008), Ion-mediated nucleation as an important global source of tropospheric aerosols, Atmos. Chem. Phys. 8, 2537-2554, doi:10.5194/acp-8-2537-2008.

F. Rohrer, ICG-2: Troposphere, Forschungszentrum Jülich, D-52425 Jülich, Germany.

S. Rohs, C. Schiller, R. Spang, and H. Vos, ICG-1: Stratosphere, Forschungszentrum Jülich, D-52425 Jülich, Germany. (s.rohs@fz-juelich.de) 